MATHEMATICS OF COMPUTATION

Volume 78, Number 266, April 2009, Pages 969-997

S 0025-5718(08)02195-9

Article electronically published on October 27, 2008

\title{
STIELTJES-TYPE POLYNOMIALS ON THE UNIT CIRCLE
}

\author{
B. DE LA CALLE YSERN, G. LÓPEZ LAGOMASINO, AND L. REICHEL
}

\begin{abstract}
Stieltjes-type polynomials corresponding to measures supported on the unit circle $\mathbb{T}$ are introduced and their asymptotic properties away from $\mathbb{T}$ are studied for general classes of measures. As an application, we prove the convergence of an associated sequence of interpolating rational functions to the corresponding Carathéodory function. In turn, this is used to give an estimate of the rate of convergence of certain quadrature formulae that resemble the Gauss-Kronrod rule, provided that the integrand is analytic in a neighborhood of $\mathbb{T}$.
\end{abstract}

\section{INTRODUCTION}

Let $\mu$ be a finite positive Borel measure on the real line $\mathbb{R}$ whose compact support contains infinitely many points. Let $p_{n}(x)=\gamma_{n} x^{n}+\ldots, \gamma_{n}>0$, denote the orthonormal polynomial of degree $n$ with respect to $\mu$; i.e.,

$$
\int p_{n}(x) p_{m}(x) d \mu(x)=\delta_{n, m} .
$$

The $n$th function of the second kind associated with $\mu$ is defined by

$$
q_{n}(z)=\int \frac{p_{n}(x)}{z-x} d \mu(x) .
$$

Note that, by (1) $q_{n}(z)=\mathcal{O}\left(1 / z^{n+1}\right), z \rightarrow \infty$. Therefore,

$$
\frac{1}{q_{n}(z)}=\gamma_{n} E_{n+1}(z)+\mathcal{O}(1 / z), \quad z \rightarrow \infty
$$

where $E_{n+1}$ is a monic polynomial of degree $n+1$. The polynomials $E_{n+1}$ were introduced by Stieltjes [12] in 1894 for the case of the Legendre measure $d \mu(x)=d x$ and are usually named after him. It is easy to see that they satisfy the orthogonality relations

$$
\int x^{k} E_{n+1}(x) p_{n}(x) d \mu(x)=0, \quad k=0,1, \ldots, n .
$$

Received by the editor October 3, 2007 and, in revised form, April 25, 2008.

2000 Mathematics Subject Classification. Primary 65D32, 42A10, 42C05; Secondary 30E20.

Key words and phrases. Quadrature rules on the unit circle, para-orthogonal polynomials, Stieltjes polynomials, Gauss-Kronrod quadrature.

The work of B. de la Calle received support from Dirección General de Investigación (DGI), Ministerio de Educación y Ciencia, under grants MTM2006-13000-C03-02 and MTM2006-07186 and from UPM-CAM under grants CCG07-UPM/000-1652 and CCG07-UPM/ESP-1896.

The work of G. López was supported by DGI under grant MTM2006-13000-C03-02 and by UC3M-CAM through CCG06-UC3M/ESP-0690.

The work of L. Reichel was supported by an OBR Research Challenge Grant.

(C)2008 American Mathematical Society Reverts to public domain 28 years from publication 
Since their introduction, and especially in the last four decades, Stieltjes polynomials have attracted a great deal of attention coming from a twofold origin. On one hand, though they are orthogonal with respect to the real measure $p_{n}(x) d \mu(x)$, their zeros occasionally show similar properties to those of classical orthogonal polynomials. The ultraspherical measures

$$
d \mu(x)=\left(1-x^{2}\right)^{\lambda-1 / 2} d x, \quad \lambda>-1 / 2,
$$

suffice to display the rich behavior of the zeros of $E_{n+1}$. Depending on $\lambda$, they may have exemplary zeros which are real, simple, and interlace the zeros of the corresponding orthogonal polynomial, or erratic when most of the zeros are complex (see [27, 21, 5]).

On the other hand, a renewed interest in Stieltjes polynomials is motivated by the use of their zeros as additional nodes in the so-called Gauss-Kronrod quadrature formulae introduced by Kronrod [15] in the sixties. Such quadrature rules arose from the need of estimating simultaneously an approximate value of the integral and the error as well. These rules take the form

$$
\int f(x) d \mu(x)=\sum_{i=1}^{n} A_{n, i} f\left(x_{n, i}\right)+\sum_{j=1}^{n+1} B_{n, j} f\left(y_{n, j}\right)+R_{2 n+1}(f),
$$

where $\left\{x_{n, i}\right\}_{i=1}^{n}$ are the zeros of $p_{n}$, i.e., the Gaussian nodes. The remaining nodes $\left\{y_{n, j}\right\}_{j=1}^{n+1}$ and the quadrature weights $\left\{A_{n, i}\right\}_{i=1}^{n}$ and $\left\{B_{n, j}\right\}_{j=1}^{n+1}$ are chosen so that the rule has the highest possible degree of polynomial exactness. This requirement is equivalent to the fact that the nodal polynomial

$$
\prod_{j=1}^{n+1}\left(x-y_{n, j}\right)
$$

satisfies the orthogonality relations (3). Hence, the nodes $\left\{y_{n, j}\right\}_{j=1}^{n+1}$ turn out to be the zeros of the Stieltjes polynomial $E_{n+1}$.

Gauss-Kronrod rules can be computed efficiently [1, 6, 16] and are used in packages for automatic integration 22. Additionally, the zeros of the Stieltjes polynomials have proved to be useful in product integration [7] and interpolation [8]. For further details on Stieltjes polynomials and Gauss-Kronrod quadrature formulae; see the surveys [9, 18, 19.

One of the aims of this paper is to define polynomials analogous to the Stieltjes polynomials for measures supported on the unit circle $\mathbb{T}$. This is done in Section 2 As will be seen in the next two sections, the resulting polynomial, called of Stieltjestype, has some distinctive features but shares with its counterpart on the real line two essential properties; namely, its special relationship with the corresponding function of the second kind and satisfying orthogonality conditions which allow its zeros to be considered optimal additional nodes to the Szegö quadrature (the analog on $\mathbb{T}$ of the Gauss-Jacobi rule). Stieltjes-type polynomials are not required to have their zeros lying on the support of the measure since this property is not fulfilled by all Stieltjes polynomials on the real line.

The rest of the paper is organized as follows. Section 3 contains some auxiliary material; in particular, an integral representation which is the key for subsequent developments. Section 4 is mainly dedicated to studying the asymptotic behavior of the second type functions. This is used in Section 5 to establish asymptotic properties of the Stieltjes-type polynomials for general classes of measures. In 
particular, we prove that their zeros approach $\mathbb{T}$ as the degree of the polynomials goes to infinity and find the zero limit distribution. Finally, in the last section, we prove the convergence of a related sequence of interpolating rational functions to the Carathéodory function defined by the measure. This is used to obtain an estimate of the rate of convergence of the quadrature formulae given by the Szegö and Stieltjes-type nodes provided that the integrand is analytic in a neighborhood of $\mathbb{T}$. We refer to these quadrature formulae as Szegö-Kronrod rules. They are well suited for the integration of periodic functions with known periodicity. The present paper provides the foundation for these quadrature rules. For Stieltjes polynomials on the real line, similar properties to those appearing in this paper have been shown in 2 .

\section{Stieltues-TyPe POLYNOMials}

Let $\Phi_{n}$ and $\varphi_{n}$ be the monic orthogonal and orthonormal polynomials, respectively, of degree $n$ with respect to a finite positive Borel measure $\sigma$, whose support $S(\sigma)$ is contained on the unit circle $\mathbb{T}$ and has infinitely many points. Thus, $\varphi_{n}=\kappa_{n} \Phi_{n}=\Phi_{n} /\left\|\Phi_{n}\right\|$, where

$$
\langle f, g\rangle=\int f(\zeta) \overline{g(\zeta)} d \sigma(\zeta), \quad\|f\|^{2}=\langle f, f\rangle .
$$

Let $\mathbb{D}=\{z \in \mathbb{C}:|z|<1\}$. The polynomials $\Phi_{n}$ have all their zeros in $\mathbb{D}$ so they cannot be used to construct on $\mathbb{T}$ an analog of the Gauss-Jacobi rule. Instead, polynomials called para-orthogonal (because of deficiencies in their orthogonality properties) are considered (see [11, 14]). Though the introduction of para-orthogonal polynomials is generally traced back to [14, we have noticed that they appear previously in Theorem III of [10] by Ya. L. Geronimus.

A polynomial $W_{n}$ of degree $n$ is said to be para-orthogonal with respect to the measure $\sigma$ if it satisfies the conditions

$$
\left\langle W_{n}, z^{k}\right\rangle=0, \quad k=1, \ldots, n-1 ; \quad\left\langle W_{n}, 1\right\rangle \neq 0 \neq\left\langle W_{n}, z^{n}\right\rangle
$$

$$
W_{n}^{*}=\beta W_{n}, \quad|\beta|=1 .
$$

For each $n$ such polynomials exist. It is well known that their zeros are simple and lie on the unit circle $\mathbb{T}$. Moreover (cf. [14, Theorem 6.1), $W_{n}$ is para-orthogonal with respect to $\sigma$ if and only if $W_{n}(z)=\xi_{n}\left(\varphi_{n}+\tau_{n} \varphi_{n}^{*}\right), \tau_{n} \in \mathbb{T}, \xi_{n} \in \mathbb{C} \backslash\{0\}$, and $\varphi_{n}^{*}(z)=z^{n} \overline{\varphi_{n}(1 / \bar{z})}$. In the sequel, we let $w_{n}=\varphi_{n}+\tau_{n} \varphi_{n}^{*}$, and write $W_{n}$ for monic para-orthogonal polynomials. Clearly, $w_{n}$ depends on $\tau_{n}$ but this omission will not lead to confusion.

Throughout this paper $\mathcal{P}_{n}$ denotes the space of all polynomials of degree less than or equal to $n$ and $\Lambda_{p, q}, p, q \geq 0$, is the space of all rational functions of the form $h / z^{p}, h \in \mathcal{P}_{p+q}$. This notation differs from the one usually employed.

When approximating the integral

$$
I_{\sigma}(f)=\int_{\mathbb{T}} f(\zeta) d \sigma(\zeta)
$$

by means of a quadrature rule

$$
I_{n}(f)=\sum_{i=1}^{n} \lambda_{n, i} f\left(z_{n, i}\right),
$$


it is natural to require that $I_{n}$ be exact in a space $\Lambda_{p, q}, p+q \geq n-1$. It turns out (cf. [14]) that if the nodes $\left\{z_{n, i}\right\}_{i=1}^{n}$ are chosen to be the zeros of a para-orthogonal polynomial $W_{n}$, there exist positive numbers $\left\{\lambda_{n, i}\right\}_{i=1}^{n}$ such that $I_{\sigma}(f)=I_{n}(f), f \in$ $\Lambda_{n-1, n-1}$. Such quadrature formulae are called Szegó rules. It can be shown that the space $\Lambda_{n-1, n-1}$ is the largest possible space of exactness of type $\Lambda_{p, q}$ for any rule $I_{n}$, provided that the $n$ nodes belong to $\mathbb{T}$.

Now, we want to construct a quadrature rule retaining the Szegö nodes and adding $m$ new nodes in an optimal way. The new nodes and all the quadrature weights are chosen so that the quadrature rule is exact in the largest space $\Lambda_{p, q}$. The number $m$ will be the smallest natural number such that $\Lambda_{p, q} \supseteq \Lambda_{n-1, n-1}$. The new nodes cannot be equal to 0 , since the rational functions in $\Lambda_{p, q}$ are to be evaluated at these points.

In order to properly characterize those optimal additional nodes, we will need some preliminary results on interpolatory rules with possibly repeated nodes. Given the nodal polynomial

$$
P_{N}(z)=\prod_{i=1}^{M}\left(z-z_{i}\right)^{\alpha_{i}}, \quad \alpha_{1}+\cdots+\alpha_{M}=N, \quad z_{i} \in \mathbb{C} \backslash\{0\},
$$

where $z_{i} \neq z_{j}$ whenever $i \neq j$, we consider the following generalized quadrature formulae

$$
I_{N}(f)=\sum_{i=1}^{M} \sum_{j=0}^{\alpha_{i}-1} \mu_{i, j} f^{(j)}\left(z_{i}\right)
$$

where $f^{(j)}$ denotes the $j$ th derivative of $f$. Such a rule is said to be interpolatory if it is exact in $\Lambda_{p, q}$ with $p+q \geq N-1$; that is, $I_{\sigma}(L)=I_{N}(L), L \in \Lambda_{p, q}$.

Proposition 1. Let $P_{N}$ be the polynomial given by (5) and let $p$ and $q$ be nonnegative integers with $p+q=N-1$. Then, there exists a unique system of complex numbers $\left\{\mu_{i, j}\right\}, i=1, \ldots, M, j=0, \ldots, \alpha_{i}-1$, such that the quadrature rule (6) is exact in $\Lambda_{p, q}$.

Proof. Following standard arguments of Hermite interpolation (see, for instance, [3, Chapter 2, Section 11.2), given any $i=1, \ldots, M$ and $j=0, \ldots, \alpha_{i}-1$, set

$$
R_{i, j}(z)=\frac{P_{N}(z)}{z^{p}\left(z-z_{i}\right)^{\alpha_{i}}} \frac{\left(z-z_{i}\right)^{j}}{j !} \sum_{k=0}^{\alpha_{i}-j-1} A_{i, k} \frac{\left(z-z_{i}\right)^{k}}{k !},
$$

where

$$
A_{i, k}=\left(\frac{z^{p}\left(z-z_{i}\right)^{\alpha_{i}}}{P_{N}(z)}\right)^{(k)}\left(z_{i}\right)
$$

It is clear that $R_{i, j} \in \Lambda_{p, q}$ and not difficult to verify that

$$
R_{i, j}^{(m)}\left(z_{k}\right)=\delta_{i, k} \delta_{j, m},
$$

where $\delta_{j, m}$ denotes the Kronecker delta.

For any function $f$ for which the right-hand side of (6) makes sense, there exists a unique element of $\Lambda_{p, q}$ defined by

$$
R_{f}(z)=\sum_{i=1}^{M} \sum_{j=0}^{\alpha_{i}-1} f^{(j)}\left(z_{i}\right) R_{i, j}(z)
$$


such that

$$
R_{f}^{(j)}\left(z_{i}\right)=f^{(j)}\left(z_{i}\right), \quad i=1, \ldots, M ; \quad j=0, \ldots, \alpha_{i}-1 .
$$

Now, take $\mu_{i, j}=I_{\sigma}\left(R_{i, j}\right)$. Then $I_{N}(f)=I_{\sigma}\left(R_{f}\right)$. In particular, for any $L \in \Lambda_{p, q}$ we obtain that $I_{N}(L)=I_{\sigma}\left(R_{L}\right)=I_{\sigma}(L)$ since $R_{L}=L$.

Concerning uniqueness, suppose that there exists another system of complex numbers $\left\{\mu_{i, j}^{\prime}\right\}, i=1, \ldots, M, j=0, \ldots, \alpha_{i}-1$, such that (6) is exact in $\Lambda_{p, q}$. Since $R_{i, j} \in \Lambda_{p, q}$, then because of (77), $I_{\sigma}\left(R_{i, j}\right)=I_{N}\left(R_{i, j}\right)=\mu_{i, j}^{\prime}$.

If $I_{N}$ is exact in a larger space $\Lambda_{r, s}, r+s \geq N$, then the nodal polynomial must satisfy certain orthogonality relations as indicated in the following proposition.

Proposition 2. Let $I_{N}$ be a quadrature rule given by (6) with nodal polynomial (5). Let $p$ and $q$ be non-negative integers with $p+q=N-1$. Then

$$
I_{N} \text { is exact in } \Lambda_{r, s} \supset \Lambda_{p, q} \Longleftrightarrow\left\{\begin{array}{l}
(i) I_{N} \text { is exact in } \Lambda_{p, q}, \\
(i i)\left\langle P_{N}(z), z^{k}\right\rangle=0, k=N-s, \ldots, r .
\end{array}\right.
$$

Proof. If $I_{N}$ is exact in $\Lambda_{r, s} \supset \Lambda_{p, q}$, then (i) is trivial. As for (ii), note that $\Lambda_{r, s}=\Lambda_{p, q}$ would imply $N-s>r$ and there would be nothing to prove. Otherwise, fix $k=N-s, \ldots, r$, and consider $P_{N}(z) / z^{k} \in \Lambda_{k, N-k} \subset \Lambda_{r, s}$. Then

$$
\left\langle P_{N}(z), z^{k}\right\rangle=I_{\sigma}\left(\frac{P_{N}(z)}{z^{k}}\right)=I_{N}\left(\frac{P_{N}(z)}{z^{k}}\right)=0,
$$

since the polynomial $P_{N}$ and its derivatives are evaluated at their zeros.

Conversely, let $L \in \Lambda_{r, s}$ and denote by $R_{L}$ the unique element of $\Lambda_{p, q}$ interpolating $L$ at the zeros of $P_{N}$. We obtain

$$
I_{N}(L)=I_{N}\left(R_{L}\right)=I_{\sigma}\left(R_{L}\right),
$$

because of (i). On the other hand, the polynomial $\left(L-R_{L}\right) z^{r} \in \mathcal{P}_{r+s}$ and vanishes at the zeros of $P_{N}$. Thus, $\left(L-R_{L}\right) z^{r}=P_{N}(z) T(z)$, where $T \in \mathcal{P}_{r+s-N}$. It follows that

$$
I_{\sigma}\left(L-R_{L}\right)=I_{\sigma}\left(P_{N}(z) \frac{T(z)}{z^{r}}\right)=\left\langle P_{N}(z), z^{r} \overline{T(z)}\right\rangle=0,
$$

due to (ii), which together with (8) proves the result.

The next proposition shows that in order to increase the degree of exactness of a Szegö rule with $n$ nodes, we must add at least $n$ new nodes.

Proposition 3. Let $W_{n}$ be a monic para-orthogonal polynomial of degree $n$ with respect to the measure $\sigma$ and let $T_{m}$ be a monic polynomial of degree $m \in \mathbb{N}$ with $T_{m}(0) \neq 0$. Let $I_{n+m}$ be a quadrature rule with nodal polynomial $W_{n} T_{m}$, and assume that $I_{n+m}$ is exact in $\Lambda_{r, s} \nsupseteq \Lambda_{n-1, n-1}$. Then, $m \geq n$.

Proof. We will reason by contradiction. Suppose that $m \leq n-1$. Then $I_{n+m}$ is exact in $\Lambda_{r, s} \supset \Lambda_{n-1, n-1} \supset \Lambda_{n-1, m}$. Therefore, we can apply Proposition 2 with $N=n+m$ and $P_{N}=W_{n} T_{m}$ to obtain

$$
\left\langle W_{n} T_{m}, z^{k}\right\rangle=0, \quad k=m+n-s, \ldots, r .
$$


Since $\Lambda_{r, s} \nsupseteq \Lambda_{n-1, n-1}$, either $r \geq n$ or $s \geq n$. If $r \geq n$, then $T_{m}(z)=z p_{m-1}+a$ with $a \neq 0$ and $p_{m-1} \in \mathcal{P}_{m-1}$. It follows from (9) and (4) that

$$
\begin{aligned}
0 & =\left\langle W_{n} T_{m}, z^{n}\right\rangle=\left\langle W_{n} z p_{m-1}(z), z^{n}\right\rangle+\left\langle W_{n} a, z^{n}\right\rangle \\
& =\left\langle W_{n}, z^{n-1} \overline{p_{m-1}(z)}\right\rangle+a\left\langle W_{n}, z^{n}\right\rangle=a\left\langle W_{n}, z^{n}\right\rangle,
\end{aligned}
$$

which contradicts (4). In the case $s \geq n$, we can write $T_{m}(z)=z^{m}+q_{m-1}$ with $q_{m-1} \in \mathcal{P}_{m-1}$ and, using again (9) and (4), we have

$$
\begin{aligned}
0 & =\left\langle W_{n} T_{m}, z^{m}\right\rangle=\left\langle W_{n} z^{m}, z^{m}\right\rangle+\left\langle W_{n} q_{m-1}(z), z^{m}\right\rangle \\
& =\left\langle W_{n}, 1\right\rangle+\left\langle W_{n}, \overline{q_{m-1}(z)} z^{m}\right\rangle=\left\langle W_{n}, 1\right\rangle,
\end{aligned}
$$

which also contradicts (4). Consequently, $m \geq n$ as we wanted to prove.

We are in a position to define the Stieltjes-type polynomials based on the orthogonality conditions (9) for $m=n$. For convenience, we will write those relations in an equivalent form. Fix $n \in \mathbb{N}, \tau_{n} \in \mathbb{T}$, and $m \in\{0,1, \ldots, n-1\}$. Let $S_{n, m}$ be the monic polynomial of least degree, such that

$$
\left\langle S_{n, m} z^{\nu-m}, w_{n}\right\rangle=0, \quad \nu=0,1, \ldots, n-1,
$$

and set $s_{n, m}=S_{n, m} /\left\|\Phi_{n}\right\|=\kappa_{n} S_{n, m}$. Finding $S_{n, m}$ reduces to solving a homogeneous system of $n$ equations with $n+1$ unknowns (thus non-trivial solutions exist). We take any non-trivial solution of least degree and normalize it to have leading coefficient one. It is easy to see that $S_{n, m}$ is uniquely determined (for $\tau_{n}$ fixed). We say that $S_{n, m}$ is the $(n, m)$ monic Stieltjes-type polynomial with respect to $\sigma$ and call $s_{n, m}$ the $(n, m)$ orthonormal Stieltjes-type polynomial (though it is not orthonormal in the usual sense). The polynomial $S_{n, m}$ also depends on $\tau_{n}$, but we will not explicitly indicate this dependence.

Alternatively, we could have defined an $(n, m)$ Laurent-Stieltjes polynomial as the element of $\Lambda_{n, m}$ given by $S_{n, m} z^{-m}$ orthogonal to a subspace not depending on $m$. However, we find it more convenient to work with algebraic polynomials than with Laurent polynomials since $m$ will eventually depend on $n$.

Lemma 1. For each $n \in \mathbb{N}, \operatorname{deg} S_{n, m}=n$ and $S_{n, m}(0)=-\tau_{n} \neq 0$.

Proof. Recall that $w_{n}=\varphi_{n}+\tau_{n} \varphi_{n}^{*}, \tau_{n} \in \mathbb{T}$. Suppose that $\operatorname{deg} S_{n, m}=k<n$. First, let us consider the case when $k>m$. We have: a) $n \geq n-k \geq 1$, b) $m+n-k \leq n-1$, and c) $\operatorname{deg} z^{n-k} S_{n, m}=n$. Using the orthogonality relations satisfied by $S_{n, m}$ and $w_{n}$, it follows that

$$
\begin{aligned}
0 & =\int \zeta^{m+n-k} \frac{S_{n, m}(\zeta)}{\zeta^{m}} \overline{w_{n}(\zeta)} d \sigma(\zeta)=\int \zeta^{n-k} S_{n, m}(\zeta) \overline{\varphi_{n}(\zeta)} d \sigma(\zeta) \\
& =\left\|\Phi_{n}\right\| \int\left|\varphi_{n}(\zeta)\right|^{2} d \sigma(\zeta)=\left\|\Phi_{n}\right\| \neq 0,
\end{aligned}
$$

which is absurd. Thus, $k<n$ only if $k \leq m$. Write $S_{n, m}(z)=a_{k_{0}} z^{k_{0}}+\cdots+z^{k}, a_{k_{0}} \neq$ 0 . Obviously, $0 \leq m-k_{0}$. Using again the orthogonality relations satisfied by $S_{n, m}$ and $w_{n}$, we have

$$
0=\int \zeta^{m-k_{0}} \frac{S_{n, m}(\zeta)}{\zeta^{m}} \overline{w_{n}(\zeta)} d \sigma(\zeta)=a_{k_{0}} \overline{\tau_{n}} \int \overline{\varphi_{n}^{*}(\zeta)} d \sigma(\zeta)=a_{k_{0}} \overline{\tau_{n}}\left\|\Phi_{n}\right\| \neq 0,
$$

which is also absurd. Therefore, $\operatorname{deg} S_{n, m}=n$ as stated. 
From (10) with $\nu=m$, writing $S_{n, m}(z)=z^{n}+\cdots+S_{n, m}(0)$, we obtain

$$
\begin{aligned}
0 & =\int S_{n, m}(\zeta) \overline{\varphi_{n}(\zeta)} d \sigma(\zeta)+\overline{\tau_{n}} \int S_{n, m}(\zeta) \overline{\varphi_{n}^{*}(\zeta)} d \sigma(\zeta) \\
& =\left\|\Phi_{n}\right\|\left(1+\overline{\tau_{n}} S_{n, m}(0)\right),
\end{aligned}
$$

that is, $\overline{\tau_{n}} S_{n, m}(0)=-1$ as we wanted to prove.

We are ready for the proof of the next result.

Theorem 1. For $n \in \mathbb{N}$ and $m=0,1, \ldots, n-1$, the interpolatory quadrature rule $I_{2 n}$ with nodal polynomial $W_{n} S_{n, m}$ is exact in $\Lambda_{n+m, 2 n-m-1}$.

Proof. Since $\operatorname{deg} S_{n, m}=n$ and $S_{n, m}(0) \neq 0$, we can apply Proposition 2 with $N=2 n$ and $P_{N}=W_{n} S_{n, m}$. Additionally, for $\nu=0,1, \ldots, n-1$, we know that

$$
\begin{aligned}
0 & =\left\langle S_{n, m} z^{\nu-m}, w_{n}\right\rangle=\overline{\tau_{n}}\left\langle S_{n, m} z^{\nu-m}, w_{n}^{*}\right\rangle=\overline{\tau_{n}}\left\langle S_{n, m} \overline{w_{n}^{*}}, z^{m-\nu}\right\rangle \\
& =\overline{\tau_{n}}\left\langle S_{n, m} w_{n} z^{-n}, z^{m-\nu}\right\rangle=\overline{\tau_{n}}\left\langle S_{n, m} w_{n}, z^{n+m-\nu}\right\rangle .
\end{aligned}
$$

Then, the defining conditions of $S_{n, m}$ are precisely the relations appearing in part (ii) of Proposition 2 with $r=n+m$ and $s=2 n-m-1$, which completes the proof.

Notice that we have excluded from the definition of the Stieltjes-type polynomials the cases for which the rule $I_{2 n}$ would be exact in $\Lambda_{n-1,2 n}$ and $\Lambda_{2 n, n-1}$, corresponding to $m=-1$ and $m=n$, respectively. It is easy to see that it is not possible to define in general such quadrature rules. In fact, take $d \sigma(\zeta)=d \theta /(2 \pi), \zeta=\exp (i \theta)$. Then, $w_{n}(z)=z^{n}+\tau_{n}$ and the solution of (10) for $m=-1$ is the polynomial $p(z)=z^{n}$ which takes the value 0 at $z=0$, whereas the solution of (10) for $m=n$ is $p(z) \equiv 1$. The convergence of the quadrature rules $I_{2 n}$ for analytic integrands is discussed in Section 6 .

\section{AuXiliary ReSUlts}

The following integral formulas follow easily from the defining orthogonality relations satisfied by the polynomials involved.

Lemma 2. Let $z \notin \mathbb{T}$. Then the following properties hold.

For all $p \in \mathcal{P}_{n}$,

$$
p(z) \int \frac{\overline{\varphi_{n}(\zeta)}}{z-\zeta} d \sigma(\zeta)=\int \frac{p(\zeta) \overline{\varphi_{n}(\zeta)}}{z-\zeta} d \sigma(\zeta)
$$

For all $p \in \mathcal{P}_{n+1}$,

$$
p(z) \int \frac{\overline{\varphi_{n}^{*}(\zeta)}}{1-\zeta / z} d \sigma(\zeta)=\int \frac{p(\zeta) \overline{\varphi_{n}^{*}(\zeta)}}{1-\zeta / z} d \sigma(\zeta)+\left\|\Phi_{n}\right\|(p(z)-p(0)) .
$$

For all $p \in \mathcal{P}_{n}$,

$$
p(z) \int \frac{\overline{w_{n}(\zeta)}}{1-\zeta / z} d \sigma(\zeta)=\int \frac{p(\zeta) \overline{w_{n}(\zeta)}}{1-\zeta / z} d \sigma(\zeta)+\overline{\tau_{n}}\left\|\Phi_{n}\right\|(p(z)-p(0)) .
$$

For all $L \in \Lambda_{m, n-m}, m \in\{0,1, \ldots, n-1\}$,

$$
L(z) \int \frac{s_{n, m}(\zeta) \overline{w_{n}(\zeta)}}{z-\zeta} d \sigma(\zeta)=\int \frac{L(\zeta) s_{n, m}(\zeta) \overline{w_{n}(\zeta)}}{z-\zeta} d \sigma(\zeta)
$$


Proof. From orthogonality, for any $p \in \mathcal{P}_{n}$, we have

$$
\int \frac{p(z)-p(\zeta)}{z-\zeta} \overline{\varphi_{n}(\zeta)} d \sigma(\zeta)=0
$$

The property (11) follows directly by dividing the integral into two parts.

Recall that $\left\langle 1, \varphi_{n}^{*}\right\rangle=\left\langle\varphi_{n}, z^{n}\right\rangle=\left\|\Phi_{n}\right\|$ and, for $\nu=1, \ldots, n,\left\langle z^{\nu}, \varphi_{n}^{*}\right\rangle=0$. Let $p(z)=\sum_{k=0}^{n+1} a_{k} z^{k}$. Then

$$
\begin{aligned}
& \int \frac{p(z)-p(\zeta)}{z-\zeta} \overline{\varphi_{n}^{*}(\zeta)} d \sigma(\zeta)=\sum_{k=0}^{n+1} a_{k} \int \frac{z^{k}-\zeta^{k}}{z-\zeta} \overline{\varphi_{n}^{*}(\zeta)} d \sigma(\zeta) \\
& =\sum_{k=1}^{n+1} a_{k} \int\left(z^{k-1}+z^{k-2} \zeta+\cdots+\zeta^{k-1}\right) \overline{\varphi_{n}^{*}(\zeta)} d \sigma(\zeta) \\
& =\sum_{k=1}^{n+1} a_{k} z^{k-1} \int \overline{\varphi_{n}^{*}(\zeta)} d \sigma(\zeta)=\left\|\Phi_{n}\right\| \frac{p(z)-p(0)}{z} .
\end{aligned}
$$

Multiplying this equality by $z$ and rewriting it conveniently yields (12).

The next relation is obtained similarly. Let $p(z)=\sum_{k=0}^{n} a_{k} z^{k}$. Then

$$
\begin{aligned}
\int \frac{p(z)-p(\zeta)}{z-\zeta} \overline{w_{n}(\zeta)} d \sigma(\zeta) & =\sum_{k=1}^{n} a_{k} z^{k-1} \int \overline{\varphi_{n}(\zeta)+\tau_{n} \varphi_{n}^{*}(\zeta)} d \sigma(\zeta) \\
& =\overline{\tau_{n}} \frac{p(z)-p(0)}{z}\left\|\Phi_{n}\right\|,
\end{aligned}
$$

which is equivalent to (13).

If $L \in \Lambda_{m, n-m}$, then $L(z)=p(z) / z^{m}$, where $p \in \mathcal{P}_{n}$. Therefore,

$$
\frac{L(z)-L(\zeta)}{z-\zeta}=\frac{\zeta^{m} p(z)-z^{m} p(\zeta)}{(z-\zeta) z^{m} \zeta^{m}}
$$

Since $\left(\zeta^{m} p(z)-z^{m} p(\zeta)\right) /(z-\zeta)$ is a polynomial in $\zeta$ of degree at most $n-1$, the orthogonality relations satisfied by $s_{n, m}$ yield

$$
\int \frac{L(z)-L(\zeta)}{z-\zeta} s_{n, m}(\zeta) \overline{w_{n}(\zeta)} d \sigma(\zeta)=0,
$$

which is equivalent to (14).

Applying (13) with $p(z)=z^{k}, k=1, \ldots, n$, we obtain

$$
g_{n}(z):=\int \frac{\overline{w_{n}(\zeta)}}{1-\zeta / z} d \sigma(\zeta)-\overline{\tau_{n}}\left\|\Phi_{n}\right\|=\frac{1}{z^{k}} \int \frac{\zeta^{k} \overline{w_{n}(\zeta)}}{1-\zeta / z} d \sigma(\zeta) .
$$

We refer to $g_{n}$ as the $n$th second type function associated with $\sigma$ and $w_{n}$, and will investigate some of its properties.

Lemma 3. For $z \notin \mathbb{T}$, we have

$$
g_{n}(z)=\frac{\overline{\tau_{n}}}{z^{n}} \int \frac{w_{n}(\zeta)}{1-\zeta / z} d \sigma(\zeta), \quad \overline{g_{n}(1 / \bar{z})}=-\tau_{n} z^{n} g_{n}(z),
$$


and

$$
w_{n}(z) g_{n}(z)=\int \frac{\left|w_{n}(\zeta)\right|^{2}}{1-\zeta / z} d \sigma(\zeta)-\left(1+\overline{\tau_{n}} \Phi_{n}(0)\right)
$$

Consequently,

$$
\left|w_{n}(z) g_{n}(z)\right| \leq \begin{cases}\left|1+\overline{\tau_{n}} \Phi_{n}(0)\right|\left(1+\frac{2}{1-1 /|z|}\right), & |z|>1, \\ \left|1+\overline{\tau_{n}} \Phi_{n}(0)\right|\left(1+\frac{2}{1-|z|}\right), & |z|<1,\end{cases}
$$

and

$$
\left|w_{n}(z) g_{n}(z)\right| \geq \begin{cases}\left(1+\Re\left\{\overline{\tau_{n}} \Phi_{n}(0)\right\}\right)\left(\frac{2}{1+1 /|z|}-1\right), & |z|>1, \\ \left(1+\Re\left\{\overline{\tau_{n}} \Phi_{n}(0)\right\}\right)\left(1-\frac{2}{1+1 /|z|}\right), & |z|<1 .\end{cases}
$$

In particular, $g_{n}(z) \neq 0$ for $|z| \neq 1$. If $\lim _{n \rightarrow \infty}\left(1+\Re\left\{\overline{\tau_{n}} \Phi_{n}(0)\right\}\right)^{1 / n}=1$, then

$$
\lim _{n \rightarrow \infty}\left|w_{n}(z) g_{n}(z)\right|^{1 / n}=1 \text {, }
$$

uniformly on compact subsets of $\overline{\mathbb{C}} \backslash \mathbb{T}$, where $\overline{\mathbb{C}}=\mathbb{C} \cup\{\infty\}$.

Proof. Using (15) with $k=n$, it follows that

$$
g_{n}(z)=\frac{1}{z^{n}} \int \frac{\zeta^{n} \overline{w_{n}(\zeta)}}{1-\zeta / z} d \sigma(\zeta)=\frac{\overline{\tau_{n}}}{z^{n}} \int \frac{\tau_{n} w_{n}^{*}(\zeta)}{1-\zeta / z} d \sigma(\zeta),
$$

which is the first part of (16), since $w_{n}^{*}(z)=z^{n} \overline{w_{n}(1 / \bar{z})}$ and $\tau_{n} w_{n}^{*}(z)=w_{n}(z)$. Hence,

$$
-\tau_{n} z^{n} g_{n}(z)=-\int \frac{w_{n}(\zeta)}{1-\zeta / z} d \sigma(\zeta)
$$

Using (15) with $k=1$, we have

$$
\overline{g_{n}(1 / \bar{z})}=z \int \frac{\bar{\zeta} w_{n}(\zeta)}{1-\bar{\zeta} z} d \sigma(\zeta)=-\int \frac{w_{n}(\zeta)}{1-\zeta / z} d \sigma(\zeta)
$$

and we obtain the second relation in (16).

Multiplying the first equality in (15) by $w_{n}(z)$ and using (13), formula (17) readily follows in view of the fact that $w_{n}(0) \overline{\tau_{n}}\left\|\Phi_{n}\right\|=1+\overline{\tau_{n}} \Phi_{n}(0)$.

For $|\zeta|=1$ and $|z| \neq 1$, write $\zeta / z=r e^{i \theta}$. Then

$$
\Re\left\{\frac{1}{1-\zeta / z}\right\}=\frac{1-r \cos \theta}{1-2 r \cos \theta+r^{2}}=\frac{1-r t}{1-2 r t+r^{2}}, \quad t \in[-1,1] .
$$

The function on the right-hand side increases with $t$ when $r<1$ and decreases when $r>1$. Therefore,

$$
\Re\left\{\frac{1}{1-\zeta / z}\right\} \leq \frac{1}{1+1 /|z|}<\frac{1}{2}, \quad|z|<1,
$$

and

$$
\frac{1}{2}<\frac{1}{1+1 /|z|} \leq \Re\left\{\frac{1}{1-\zeta / z}\right\}, \quad|z|>1
$$


Let $|z|>1$. It follows from (17) that

$$
\left|w_{n}(z) g_{n}(z)\right| \leq\left|1+\overline{\tau_{n}} \Phi_{n}(0)\right|+\frac{1}{1-1 /|z|} \int\left|w_{n}(\zeta)\right|^{2} d \sigma(\zeta)
$$

Direct calculations give

$$
\int\left|w_{n}(\zeta)\right|^{2} d \sigma(\zeta)=2\left(1+\Re\left\{\overline{\tau_{n}} \Phi_{n}(0)\right\}\right) .
$$

Thus,

$$
\left|w_{n}(z) g_{n}(z)\right| \leq\left|1+\overline{\tau_{n}} \Phi_{n}(0)\right|\left(1+\frac{2}{1-1 /|z|}\right), \quad|z|>1 .
$$

On the other hand,

$$
\begin{aligned}
\left|w_{n}(z) g_{n}(z)\right| & \geq\left|\Re\left\{w_{n}(z) g_{n}(z)\right\}\right| \\
& =\left.\left|\int\right| w_{n}(\zeta)\right|^{2} \Re\left\{\frac{1}{1-\zeta / z}\right\} d \sigma(\zeta)-\left(1+\Re\left\{\overline{\tau_{n}} \Phi_{n}(0)\right\}\right) \mid \\
& \geq 2 \frac{1+\Re\left\{\overline{\tau_{n}} \Phi_{n}(0)\right\}}{1+1 /|z|}-\left(1+\Re\left\{\overline{\tau_{n}} \Phi_{n}(0)\right\}\right) \\
& =\left(1+\Re\left\{\overline{\tau_{n}} \Phi_{n}(0)\right\}\right)\left(\frac{2}{1+1 /|z|}-1\right), \quad|z|>1 .
\end{aligned}
$$

For $|z|<1$, proceeding analogously, one obtains

$$
\begin{aligned}
\left|w_{n}(z) g_{n}(z)\right| & \leq \frac{|z|}{1-|z|} \int\left|w_{n}(\zeta)\right|^{2} d \sigma(\zeta)+\left|1+\overline{\tau_{n}} \Phi_{n}(0)\right| \\
& \leq\left|1+\overline{\tau_{n}} \Phi_{n}(0)\right|\left(1+\frac{2}{1-|z|}\right)
\end{aligned}
$$

and

$$
\begin{aligned}
\left|w_{n}(z) g_{n}(z)\right| & \geq\left|\Re\left\{w_{n}(z) g_{n}(z)\right\}\right| \\
& =\left.\left|\int\right| w_{n}(\zeta)\right|^{2} \Re\left\{\frac{1}{1-\zeta / z}\right\} d \sigma(\zeta)-\left(1+\Re\left\{\overline{\tau_{n}} \Phi_{n}(0)\right\}\right) \mid \\
& \geq\left(1+\Re\left\{\overline{\tau_{n}} \Phi_{n}(0)\right\}\right)-2 \frac{1+\Re\left\{\overline{\tau_{n}} \Phi_{n}(0)\right\}}{1+1 /|z|} \\
& =\left(1+\Re\left\{\overline{\tau_{n}} \Phi_{n}(0)\right\}\right)\left(1-\frac{2}{1+1 /|z|}\right) .
\end{aligned}
$$

These are the inequalities (18) and (19).

The fact that $g_{n}(z) \neq 0$ for $|z| \neq 1$ follows from the lower bounds in (19), since $\Re\left\{\overline{\tau_{n}} \Phi_{n}(0)\right\}>-1$ and the factors in parentheses are positive. The limit (20) is an immediate consequence of the inequalities in (18) and (19) under the given conditions.

The following integral formulae are the key to obtaining the asymptotic behavior of $\left\{s_{n, m}\right\}$ as $n \rightarrow \infty$ discussed in Section 5 below. 
Lemma 4. Let $n \in \mathbb{N}, m \in\{0,1, \ldots, n-1\}$. We have

$$
s_{n, m}(z) g_{n}(z)-1=\int \frac{s_{n, m}(\zeta) \overline{w_{n}(\zeta)}}{1-\zeta / z} d \sigma(\zeta), \quad|z| \neq 1 .
$$

Set $s_{n, m}=s_{n, m, 1}+s_{n, m, 2}$, where $s_{n, m, 1}$ contains the first $m+1$ terms of $s_{n, m}$. Then

$$
s_{n, m, 2}(z) g_{n}(z)-1=\frac{z^{m+1} g_{n}(z)}{2 \pi i} \int_{\gamma_{R}} \frac{d \zeta}{\zeta^{m+1} g_{n}(\zeta)(\zeta-z)}, \quad|z|>R,
$$

and

$$
s_{n, m, 1}(z) g_{n}(z)-1=\frac{z^{m+1} g_{n}(z)}{2 \pi i} \int_{\gamma_{r}} \frac{d \zeta}{\zeta^{m+1} g_{n}(\zeta)(z-\zeta)}, \quad|z|<r,
$$

where $\gamma_{R}$ is any positively oriented circle centered at the origin of radius $R>1$ and $\gamma_{r}$ is any positively oriented circle centered at the origin of radius $r<1$.

Proof. Combining the first equality in (15), formula (13) with $p=s_{n, m}$, and the last equality in Lemma 1, we obtain

$$
\begin{aligned}
s_{n, m}(z) g_{n}(z) & =\int \frac{s_{n, m}(\zeta) \overline{w_{n}(\zeta)}}{1-\zeta / z} d \sigma(\zeta)-\overline{\tau_{n}}\left\|\Phi_{n}\right\| s_{n, m}(0) \\
& =\int \frac{s_{n, m}(\zeta) \overline{w_{n}(\zeta)}}{1-\zeta / z} d \sigma(\zeta)+1,
\end{aligned}
$$

which is (21).

According to (16) $, z^{n} g_{n}(z)=\left\|\Phi_{n}\right\|(1+o(1)), z \rightarrow \infty$. On the other hand, relation (14) with $L(z)=z^{n-m}$ gives us that

$$
\int \frac{s_{n, m}(\zeta) \overline{w_{n}(\zeta)}}{1-\zeta / z} d \sigma(\zeta)=\mathcal{O}\left(1 / z^{n-m}\right), \quad z \rightarrow \infty .
$$

Consequently, $s_{n, m}(z)-1 / g_{n}(z)=\mathcal{O}\left(z^{m}\right), z \rightarrow \infty$. Therefore, we have

$$
\frac{s_{n, m}(z)-1 / g_{n}(z)}{z^{m+1}}=\mathcal{O}(1 / z), \quad z \rightarrow \infty,
$$

and the left-hand side is analytic outside the unit circle because $g_{n}(z) \neq 0$ for $|z| \neq 1$. Making use of Cauchy's integral formula and Cauchy's theorem, we obtain

$$
\begin{aligned}
\frac{s_{n, m}(z)-\frac{1}{g_{n}(z)}}{z^{m+1}} & =\frac{1}{2 \pi i} \int_{\gamma_{R}} \frac{s_{n, m}(\zeta)-1 / g_{n}(\zeta)}{\zeta^{m+1}(z-\zeta)} d \zeta \\
& =\frac{1}{2 \pi i} \int_{\gamma_{R}} \frac{s_{n, m}(\zeta)}{\zeta^{m+1}(z-\zeta)} d \zeta+\frac{1}{2 \pi i} \int_{\gamma_{R}} \frac{d \zeta}{\zeta^{m+1} g_{n}(\zeta)(\zeta-z)} \\
& =\frac{s_{n, m, 1}(z)}{z^{m+1}}+\frac{1}{2 \pi i} \int_{\gamma_{R}} \frac{d \zeta}{\zeta^{m+1} g_{n}(\zeta)(\zeta-z)} .
\end{aligned}
$$

Rearranging this formula gives (22).

In order to prove (23), let us show that the function

$$
\frac{s_{n, m}(z)-1 / g_{n}(z)}{z^{m+1}}
$$


is analytic inside the unit circle. In fact, since $g_{n}(z) \neq 0$ whenever $|z| \neq 1$, it can only have a singularity at $z=0$ which is not the case since, according to (14) with $L(z)=z^{-m}$, we know that (see also (21))

$$
\int \frac{s_{n, m}(\zeta) \overline{w_{n}(\zeta)}}{1-\zeta / z} d \sigma(\zeta)=\mathcal{O}\left(z^{m+1}\right), \quad z \rightarrow 0
$$

Applying Cauchy's integral formula and Cauchy's theorem, it follows that

$$
\begin{aligned}
\frac{s_{n, m}(z)-\frac{1}{g_{n}(z)}}{z^{m+1}} & =\frac{1}{2 \pi i} \int_{\gamma_{r}} \frac{s_{n, m}(\zeta)-1 / g_{n}(\zeta)}{\zeta^{m+1}(\zeta-z)} d \zeta \\
& =\frac{1}{2 \pi i} \int_{\gamma_{r}} \frac{s_{n, m}(\zeta)}{\zeta^{m+1}(\zeta-z)} d \zeta+\frac{1}{2 \pi i} \int_{\gamma_{r}} \frac{d \zeta}{\zeta^{m+1} g_{n}(\zeta)(z-\zeta)} \\
& =\frac{s_{n, m, 2}(z)}{z^{m+1}}+\frac{1}{2 \pi i} \int_{\gamma_{r}} \frac{d \zeta}{\zeta^{m+1} g_{n}(\zeta)(\zeta-z)}
\end{aligned}
$$

and (23) readily follows.

From the proof of Lemma 4, we obtain

$$
\begin{aligned}
1 / g_{n}(z) & =s_{n, m, 2}(z)+\mathcal{O}\left(z^{m}\right), \quad z \rightarrow \infty \\
1 / g_{n}(z) & =s_{n, m, 1}(z)+\mathcal{O}\left(z^{m+1}\right), \quad z \rightarrow 0 .
\end{aligned}
$$

Keeping in mind that $s_{n, m}=s_{n, m, 1}+s_{n, m, 2}$, relations (24) are the analog in this setting of (2) and could have been taken as the starting point for defining the polynomials $s_{n, m}$. Having two points to interpolate explains the need for the parameter $m$ and the richer structure of the Stieltjes-type polynomials when compared with the construction on the real line.

With the aid of Lemma 4, we can reduce the asymptotic behavior of the polynomials $s_{n, m}$ to that of the second type functions $g_{n}$ which are defined, independently of the first, in terms of the para-orthogonal polynomials. The asymptotic properties of para-orthogonal polynomials are easy consequences of the corresponding results for orthonormal polynomials (cf. 4]). For convenience of the reader and easy reference, we state these properties in the form of lemmas in Section 4. Nonetheless, to our knowledge, equation (26) below is new under the assumption that $\sigma \in \mathbf{R e g} \mathbb{T}$. The main part of the following section is devoted to the study of the asymptotics of the second type functions.

\section{Asymptotics of SECOND TYPE FUnCtions}

Throughout this section, we will for brevity use the notation

$$
\lim _{n \rightarrow \infty} f_{n}(z)=f(z), \quad K \subset U,
$$

which stands for uniform convergence of the sequence of functions $\left\{f_{n}\right\}, n \in \mathbb{N}$, to $f$ for $z$ varying in any given compact subset $K$ of a specified open set $U$.

Let us recall some important classes of measures. A measure $\sigma$ supported on the unit circle is said to belong to the class Reg if

$$
\lim _{n \rightarrow \infty} \kappa_{n}^{1 / n}=1 / \operatorname{cap}(S(\sigma))
$$


where $\operatorname{cap}(S(\sigma))$ denotes the logarithmic capacity of the support $S(\sigma)$ of $\sigma$. For more details on this class of measures and equivalent formulations of the defining condition; see Theorem 3.1.1 in [26]. We are interested in an important subclass, which will be denoted by $\sigma \in \mathbf{R e g} \mathbb{T}$, for which (cf. [24, Corollary 5.2.2)

$$
\lim _{n \rightarrow \infty} \kappa_{n}^{1 / n}=1=1 / \operatorname{cap}(\mathbb{T}) .
$$

In this case, the condition above is equivalent to (see [26])

$$
\lim _{n \rightarrow \infty}\left|\varphi_{n}(z)\right|^{1 / n}=|z|, \quad K \subset \mathbb{C} \backslash \overline{\mathbb{D}},
$$

where $\overline{\mathbb{D}}=\{z \in \mathbb{C}:|z| \leq 1\}$. Recall that $\mathbb{D}=\{z \in \mathbb{C}:|z|<1\}$.

Another important class of measures is that for which

$$
\lim _{n \rightarrow \infty} \Phi_{n}(0)=0 .
$$

This condition is equivalent (see Theorem 1.7.4 and the historical remark concerning this theorem on page 107 in [25, Part I) to

$$
\lim _{n \rightarrow \infty} \frac{\kappa_{n+1}}{\kappa_{n}}=1, \quad \lim _{n \rightarrow \infty} \frac{\varphi_{n+1}(z)}{\varphi_{n}(z)}=z, \quad K \subset \mathbb{C} \backslash \mathbb{D},
$$

and

$$
\lim _{n \rightarrow \infty} \frac{\varphi_{n}^{*}(z)}{\varphi_{n}(z)}=0, \quad K \subset \overline{\mathbb{C}} \backslash \overline{\mathbb{D}}
$$

(among other relations). E. A. Rakhmanov proved that if $\sigma^{\prime}>0$ almost everywhere on the unit circle, then $\lim _{n \rightarrow \infty} \Phi_{n}(0)=0$ (see [23] and the references therein for an historic background).

We also consider the Szegö class of measures. We say that $\sigma \in \mathbf{S}$ if $\log \sigma^{\prime} \in L^{1}(\mathbb{T})$. In this case (see, for example, Theorem 2.4.1 in [25], Part I)

$$
\lim _{n \rightarrow \infty} \varphi_{n}^{*}(z)=\frac{1}{S_{\sigma}(z)}, \quad K \subset \mathbb{D}
$$

which is equivalent to

$$
\lim _{n \rightarrow \infty} \frac{\varphi_{n}(z)}{z^{n}}=\frac{1}{\overline{S_{\sigma}(1 / \bar{z})}}=S_{\sigma}(z), \quad K \subset \overline{\mathbb{C}} \backslash \overline{\mathbb{D}},
$$

where

$$
S_{\sigma}(z)=\exp \left\{\frac{1}{4 \pi} \int_{\mathbb{T}} \frac{\zeta+z}{\zeta-z} \log \sigma^{\prime}(\zeta)|d \zeta|\right\}, \quad z \notin \mathbb{T} .
$$

Obviously, $\sigma \in \mathbf{S}$ implies that $\sigma^{\prime}>0$ almost everywhere on $\mathbb{T}$ which, in turn, yields $\sigma \in \operatorname{Reg} \mathbb{T}$. These classes of measures have in common that for all of them $S(\sigma)=\mathbb{T}$. Many of the results that follow may be adapted to more general classes of measures whose support is contained (strictly) in $\mathbb{T}$, but following this trail would lead us too far away from our main objectives.

Lemma 5. If $\sigma \in \boldsymbol{R e g} \mathbb{T}$, then (20) holds. Moreover,

$$
\lim _{n \rightarrow \infty}\left|w_{n}(z)\right|^{1 / n}= \begin{cases}|z|, & K \subset \mathbb{C} \backslash \overline{\mathbb{D}}, \\ 1, & K \subset \mathbb{D},\end{cases}
$$


and

$$
\lim _{n \rightarrow \infty}\left|g_{n}(z)\right|^{1 / n}= \begin{cases}1 /|z|, & K \subset \overline{\mathbb{C}} \backslash \overline{\mathbb{D}}, \\ 1, & K \subset \mathbb{D} .\end{cases}
$$

Proof. Notice that $\left|\Phi_{n}(0)\right|<1$, which implies that

$$
\limsup _{n \rightarrow \infty}\left|1+\overline{\tau_{n}} \Phi_{n}(0)\right|^{1 / n} \leq 2^{1 / n}=1 .
$$

On the other hand,

$$
\begin{aligned}
\left|1+\Re\left\{\overline{\tau_{n}} \Phi_{n}(0)\right\}\right| & \geq 1-\left|\Phi_{n}(0)\right|=\frac{1-\left|\Phi_{n}(0)\right|^{2}}{1+\left|\Phi_{n}(0)\right|} \\
& \geq \frac{1}{2}\left(1-\left|\Phi_{n}(0)\right|^{2}\right)=\frac{\kappa_{n-1}^{2}}{2 \kappa_{n}^{2}}
\end{aligned}
$$

and $\sigma \in \operatorname{Reg} \mathbb{T}$ means that $\lim _{n \rightarrow \infty} \kappa_{n}{ }^{1 / n}=1$, so we also have

$$
\liminf _{n \rightarrow \infty}\left(1+\Re\left\{\overline{\tau_{n}} \Phi_{n}(0)\right\}\right)^{1 / n}=1 .
$$

Thus, $\lim _{n \rightarrow \infty}\left(1+\Re\left\{\overline{\tau_{n}} \Phi_{n}(0)\right\}\right)^{1 / n}=1$ and (20) follows.

Let $|z|>1$. Since

$$
\frac{\left|w_{n}(z)\right|}{|z|^{n}}=\frac{\left|\varphi_{n}(z)\right|}{|z|^{n}}\left(1+\frac{\left|\Phi_{n}^{*}(z)\right|}{\left|\Phi_{n}(z)\right|}\right) \leq 2 \frac{\left|\varphi_{n}(z)\right|}{|z|^{n}}, \quad|z| \geq 1,
$$

it follows that

$$
\limsup _{n \rightarrow \infty} \frac{\left|w_{n}(z)\right|^{1 / n}}{|z|} \leq 1, \quad K \subset \overline{\mathbb{C}} \backslash \overline{\mathbb{D}} .
$$

According to (16) and the Cauchy-Schwarz inequality, we have

$$
\left|z^{n} g_{n}(z)\right| \leq \frac{2}{1-1 /|z|} \int\left|\varphi_{n}(\zeta)\right| d \sigma(\zeta) \leq \frac{2\|\sigma\|^{1 / 2}}{1-1 /|z|}, \quad|z|>1,
$$

where $\|\sigma\|$ denotes the total mass of $\sigma$. Consequently,

$$
|z| \limsup _{n \rightarrow \infty}\left|g_{n}(z)\right|^{1 / n} \leq 1, \quad K \subset \overline{\mathbb{C}} \backslash \overline{\mathbb{D}} .
$$

Combining the last inequality and (20), we get

$$
\liminf _{n \rightarrow \infty} \frac{\left|w_{n}(z)\right|^{1 / n}}{|z|}=\liminf _{n \rightarrow \infty} \frac{\left|w_{n}(z) g_{n}(z)\right|^{1 / n}}{|z|\left|g_{n}(z)\right|^{1 / n}}=\frac{\lim _{n \rightarrow \infty}\left|w_{n}(z) g_{n}(z)\right|^{1 / n}}{|z| \limsup _{n \rightarrow \infty}\left|g_{n}(z)\right|^{1 / n}} \geq 1,
$$

uniformly on compact subsets, and the first part of (26) follows. This, together with (20), gives us the first part of (27).

The second parts of (26) and (27) follow from the first ones, using the formulas $\overline{g_{n}(1 / \bar{z})}=-\tau_{n} z^{n} g_{n}(z)$ and $\tau_{n} w_{n}^{*}=w_{n}$, which we have seen before.

Lemma 6. If $\sigma$ is such that $\lim _{n \rightarrow \infty} \Phi_{n}(0)=0$, then, for all $j \in \mathbb{Z}$ and $f$ continuous on $\mathbb{T}$, it holds that

$$
\lim _{n \rightarrow \infty} \int f(z) \overline{\varphi_{n}(z)} \varphi_{n+j}^{*}(z) d \sigma(z)=0 .
$$


Consequently, for all $f$ continuous on $\mathbb{T}$, we have

$$
\lim _{n \rightarrow \infty} \int f(z)\left|w_{n}(z)\right|^{2} d \sigma(z)=\frac{1}{\pi} \int_{0}^{2 \pi} f(z)|d z| .
$$

In particular,

$$
\lim _{n \rightarrow \infty} w_{n}(z) g_{n}(z)=\left\{\begin{array}{cl}
1, & K \subset \overline{\mathbb{C}} \backslash \overline{\mathbb{D}} \\
-1, & K \subset \mathbb{D}
\end{array}\right.
$$

Proof. We first show (28). Because of the Weierstrass theorem, it suffices to show that (28) holds for $f(z)=z^{k}, k \in \mathbb{Z}$. Let $k=0$. If $j \leq-1$, then by orthogonality

$$
\int \overline{\varphi_{n}(z)} \varphi_{n+j}^{*}(z) d \sigma(z)=0,
$$

(for all sufficiently large $n$ ) which implies (28) for this value of $k$.

Let us assume that when $k=0$, (28) is true for all $j \leq l-1$ and let us show that it is also fulfilled for $j=l$. Using the recurrence formula

$$
\kappa_{n+l-1} \varphi_{n+l}^{*}(z)=\kappa_{n+l} \varphi_{n+l-1}^{*}(z)+\overline{\varphi_{n+l}(0)} z \varphi_{n+l-1}(z),
$$

we have

$$
\begin{aligned}
\int \overline{\varphi_{n}(z)} \varphi_{n+l}^{*}(z) d \sigma(z) & =\frac{\kappa_{n+l}}{\kappa_{n+l-1}} \int \overline{\varphi_{n}(z)} \varphi_{n+l-1}^{*}(z) d \sigma(z) \\
& +\overline{\Phi_{n+l}(0)} \frac{\kappa_{n+l}}{\kappa_{n+l-1}} \int z \overline{\varphi_{n}(z)} \varphi_{n+l-1}^{*}(z) d \sigma(z) .
\end{aligned}
$$

Since $\lim _{n \rightarrow \infty} \kappa_{n+l} / \kappa_{n+l-1}=1$ and $\lim _{n \rightarrow \infty} \Phi_{n}(0)=0$, using the induction hypothesis and the Cauchy-Schwarz inequality on the last integral, we obtain (28) when $j=l$. Thus, (28) is true when $k=0$.

Now, let us assume that (28) holds for all $|k| \leq l-1$ and for all $j \in \mathbb{Z}$, and let us show that it is also satisfied when $|k|=l$. For the case $k=l$, from the recurrence formula

we find that

$$
\kappa_{n-1} \varphi_{n}(z)=\kappa_{n} z \varphi_{n-1}(z)+\varphi_{n}(0) \varphi_{n-1}^{*}(z)
$$

$$
\begin{aligned}
\int z^{l} \overline{\varphi_{n}(z)} \varphi_{n+j}^{*}(z) d \sigma(z) & =\int z^{l-1} \frac{\overline{\varphi_{n}(z)}}{\bar{z}} \varphi_{n+j}^{*}(z) d \sigma(z) \\
& =\frac{\kappa_{n}}{\kappa_{n-1}} \int z^{l-1} \overline{\varphi_{n-1}(z)} \varphi_{n+j}^{*}(z) d \sigma(z) \\
& +\overline{\Phi_{n}(0)} \frac{\kappa_{n}}{\kappa_{n-1}} \int z^{l} \overline{\varphi_{n-1}^{*}(z)} \varphi_{n+j}^{*}(z) d \sigma(z) .
\end{aligned}
$$

Using the induction hypothesis and the Cauchy-Schwarz inequality, we obtain (28) when $k=l$ for all $j \in \mathbb{Z}$. The proof when $k=-l$ is analogous and we leave it to the reader. Thus, we conclude that (28) is true.

In order to prove (29), it suffices to show that for all $k \in \mathbb{Z}$,

$$
\lim _{n \rightarrow \infty} \int z^{k}\left|w_{n}(z)\right|^{2} d \sigma(z)=\frac{1}{\pi} \int_{0}^{2 \pi} z^{k}|d z|,
$$


and apply the Weierstrass theorem. Since

$$
\left|w_{n}(z)\right|^{2}=2\left|\varphi_{n}(z)\right|^{2}+2 \Re\left\{\tau_{n} \varphi_{n}^{*}(z) \overline{\varphi_{n}(z)}\right\}, \quad|z|=1,
$$

and $\lim _{n \rightarrow \infty} \Phi_{n}(0)=0$ implies that (see Theorem 5 in [17])

$$
\lim _{n \rightarrow \infty} \int z^{k}\left|\varphi_{n}(z)\right|^{2} d \sigma(z)=\frac{1}{2 \pi} \int_{0}^{2 \pi} z^{k}|d z|,
$$

formula (29) follows from (28).

According to (17) and (29),

$$
\lim _{n \rightarrow \infty} w_{n}(z) g_{n}(z)+1=\lim _{n \rightarrow \infty} \int \frac{\left|w_{n}(\zeta)\right|^{2}}{1-\zeta / z} d \sigma(\zeta)=\frac{2}{2 \pi i} \int_{\mathbb{T}} \frac{d \zeta}{\zeta(1-\zeta / z)} .
$$

Applying the residue theorem to the last integral we arrive at (30) in the sense of pointwise limit. It is easy to see that the family of functions

$$
\left\{\int \frac{\left|w_{n}(\zeta)\right|^{2}}{1-\zeta / z} d \sigma(\zeta)\right\}_{n \in \mathbb{N}}
$$

is uniformly bounded on compact subsets of $\overline{\mathbb{C}} \backslash \mathbb{T}$; therefore, the limit is uniform on compact subsets of $\overline{\mathbb{C}} \backslash \mathbb{T}$.

A result analogous to (28) appears as Proposition 9.4.4 in 25]. The author assumes that $\sigma^{\prime}>0$ a.e. on $\mathbb{T}$ and the conclusion is drawn for all $f \in L_{\infty}$. To be precise, his proof goes through continuous functions and for that part only uses the fact that $\lim _{n \rightarrow \infty} \Phi_{n}(0)=0$. As a matter of fact, under that stronger assumption, limit (29) holds for all $f \in L_{\infty}$ as well.

Lemma 7. If $\sigma$ is such that $\lim _{n \rightarrow \infty} \Phi_{n}(0)=0$, then

$$
\lim _{n \rightarrow \infty} \frac{w_{n+1}(z)}{w_{n}(z)}= \begin{cases}z, & K \subset \mathbb{C} \backslash \overline{\mathbb{D}}, \\ 1, & K \subset \mathbb{D},\end{cases}
$$

and

$$
\lim _{n \rightarrow \infty} \frac{g_{n+1}(z)}{g_{n}(z)}= \begin{cases}1 / z, & K \subset \overline{\mathbb{C}} \backslash \overline{\mathbb{D}} \\ -1, & K \subset \mathbb{D}\end{cases}
$$

Proof. We have

$$
\frac{w_{n+1}(z)}{w_{n}(z)}=\frac{\varphi_{n+1}(z)}{\varphi_{n}(z)} \frac{1+\tau_{n} \varphi_{n+1}^{*}(z) / \varphi_{n+1}(z)}{1+\tau_{n} \varphi_{n}^{*}(z) / \varphi_{n}(z)}, \quad|z|>1,
$$

and

$$
\frac{w_{n+1}(z)}{w_{n}(z)}=\frac{\varphi_{n+1}^{*}(z)}{\varphi_{n}^{*}(z)} \frac{\varphi_{n+1}(z) / \varphi_{n+1}^{*}(z)+\tau_{n}}{\varphi_{n}(z) / \varphi_{n}^{*}(z)+\tau_{n}}, \quad|z|<1 .
$$

Using the relations equivalent to $\lim _{n \rightarrow \infty} \Phi_{n}(0)=0$, (31) follows.

Since

$$
\frac{g_{n+1}(z)}{g_{n}(z)}=\frac{w_{n}(z)}{w_{n+1}(z)} \frac{w_{n+1}(z) g_{n+1}(z)}{w_{n}(z) g_{n}(z)},
$$

the second statement is a combination of the first and (30). 
Lemma 8. If $\sigma \in \mathbf{S}$, then

$$
\lim _{n \rightarrow \infty} \frac{w_{n}(z)}{z^{n}}=\frac{1}{\overline{S_{\sigma}(1 / \bar{z})}}, \quad K \subset \overline{\mathbb{C}} \backslash \overline{\mathbb{D}},
$$

and

$$
\lim _{n \rightarrow \infty} \overline{\tau_{n}} w_{n}(z)=S_{\sigma}^{-1}(z), \quad K \subset \mathbb{D} .
$$

Consequently,

$$
\lim _{n \rightarrow \infty} z^{n} g_{n}(z)=\overline{S_{\sigma}(1 / \bar{z})}=S_{\sigma}^{-1}(z), \quad K \subset \overline{\mathbb{C}} \backslash \overline{\mathbb{D}},
$$

and

$$
\lim _{n \rightarrow \infty} \tau_{n} g_{n}(z)=-S_{\sigma}(z), \quad K \subset \mathbb{D} .
$$

Proof. Notice that $\sigma \in \mathbf{S}$ implies that $\sigma^{\prime}>0$ a.e. on $\mathbb{T}$. Therefore, by Rakhmanov's theorem, $\lim _{n \rightarrow \infty} \Phi_{n}(0)=0$ and we can use all the equivalent forms of this condition. The rest of the proof is an immediate consequence of the asymptotic relations satisfied by orthonormal polynomials corresponding to measures in $\mathbf{S}$, (30), and the formulae

$$
\begin{array}{ll}
w_{n}(z)=\varphi_{n}(z)\left(1+\tau_{n} \Phi_{n}^{*}(z) / \Phi_{n}(z)\right), & |z|>1, \\
w_{n}(z)=\varphi_{n}^{*}(z)\left(\Phi_{n}(z) / \Phi_{n}^{*}(z)+\tau_{n}\right), & |z|<1 .
\end{array}
$$

\section{Asymptotics of Stieltues-type polynomials}

In the sequel, we assume that $m$ depends on $n$, i.e., $m=m_{n}$. We denote $\limsup _{n \rightarrow \infty} m_{n} / n=\bar{\rho}$ and $\liminf _{n \rightarrow \infty} m_{n} / n=\underline{\rho}$. Obviously, $0 \leq \underline{\rho} \leq \bar{\rho} \leq 1$.

Lemma 9. Let $\sigma \in \mathbf{R e g} \mathbb{T}$. Then, for each compact set $K$,

$$
\limsup _{n \rightarrow \infty}\left\|s_{n, m_{n}, 2} g_{n}-1\right\|_{K}^{1 / n} \leq\|1 / z\|_{K}^{1-\bar{\rho}}, \quad K \subset \overline{\mathbb{C}} \backslash \overline{\mathbb{D}}
$$

and

$$
\limsup _{n \rightarrow \infty}\left\|s_{n, m_{n}, 1} g_{n}-1\right\|_{K}^{1 / n} \leq\|z\| \frac{\rho}{K}, \quad K \subset \mathbb{D} .
$$

If $0<\rho \leq \bar{\rho}<1$, then for any $\varepsilon>0$ there exists $n_{0}$, such that for $n \geq n_{0}$ the zeros of $s_{n, m_{n}, 1}$ and $s_{n, m_{n}, 2}$ are in $\{z:|z| \geq 1-\varepsilon\}$ and $\{z:|z| \leq 1+\varepsilon\}$, respectively.

Proof. For brevity, we will write $m$ instead of $m_{n}$ throughout the proof. Fix a compact subset $K$ of $\mathbb{C} \backslash \overline{\mathbb{D}}$. Take the circle $\gamma_{R}$ in Lemma 4 of radius $R$ sufficiently close to 1 so that $K$ lies in the unbounded component of the complement of $\gamma_{R}$. Thus, (22) is satisfied for all $z \in K$ and

$$
\left|s_{n, m, 2}(z) g_{n}(z)-1\right| \leq C\left(\frac{|z|}{R}\right)^{m+1} \frac{\left|g_{n}(z)\right|}{\inf _{\zeta \in \gamma_{R}}\left|g_{n}(\zeta)\right|},
$$

where $C$ is a constant independent of $n$. Using (27), given $\varepsilon>0$, for all $n \geq n_{0}(\varepsilon)$, we have

$$
\left|g_{n}(z)\right| \leq \frac{1}{(|z|-\varepsilon)^{n}}, \quad \inf _{\zeta \in \gamma_{R}}\left|g_{n}(\zeta)\right| \geq \frac{1}{(R+\varepsilon)^{n}} .
$$


Therefore, for all $n \geq n_{0}(\varepsilon)$ and $z \in K$,

$$
\begin{aligned}
\left|s_{n, m, 2}(z) g_{n}(z)-1\right| & \leq C \frac{|z|^{m+1}}{(|z|-\varepsilon)^{n}} \frac{(R+\varepsilon)^{n}}{R^{m+1}} \\
& =\frac{C}{|z|^{n-m-1}(1-\varepsilon /|z|)^{n}} \frac{(R+\varepsilon)^{n}}{R^{m+1}}
\end{aligned}
$$

that is,

$$
\left\|s_{n, m, 2}(z) g_{n}(z)-1\right\|_{K} \leq C \frac{\|1 / z\|_{K}^{n-m-1}}{\left(1-\varepsilon\|1 / z\|_{K}\right)^{n}} \frac{(R+\varepsilon)^{n}}{R^{m+1}} .
$$

Taking the $n$th root and letting $n \rightarrow \infty, \varepsilon \rightarrow 0, R \rightarrow 1$, gives (37). The fact that (37) also holds on compact subsets of $\overline{\mathbb{C}} \backslash \overline{\mathbb{D}}$ follows immediately from the maximum modulus principle. The proof of (38) is analogous and we leave it to the reader.

Notice that when $0<\underline{\rho} \leq \bar{\rho}<1$, (37) and (38) yield that

$$
\lim _{n \rightarrow \infty} s_{n, m, 1}(z) g_{n}(z)=1, \quad \lim _{n \rightarrow \infty} s_{n, m, 2}(z) g_{n}(z)=1,
$$

uniformly on compact subsets of $\mathbb{D}$ and $\overline{\mathbb{C}} \backslash \overline{\mathbb{D}}$, respectively. Since $g_{n}$ has no poles in $\overline{\mathbb{C}} \backslash \mathbb{T}$, (39) and the Hurwitz theorem imply the assertion concerning the zeros of $s_{n, m, 1}$ and $s_{n, m, 2}$.

The limit behavior of the zeros of the polynomials $s_{n, m_{n}, 1}$ and $s_{n, m_{n}, 2}$ is essential in the remaining part of this paper. It is convenient to have a similar result for the limit case when $\underline{\rho}=0, \bar{\rho}=1$ even if this imposes some loss of generality.

Lemma 10. Let $\sigma \in \mathbf{S}$ and $\lim _{n \rightarrow \infty} m_{n}=\lim _{n \rightarrow \infty} n-m_{n}=\infty$ Then,

$$
\lim _{n \rightarrow \infty} s_{n, m_{n}, 2}(z) g_{n}(z)=1 \text {, }
$$

uniformly on compact subsets of $\overline{\mathbb{C}} \backslash \overline{\mathbb{D}}$ and

$$
\lim _{n \rightarrow \infty} s_{n, m_{n}, 1}(z) g_{n}(z)=1,
$$

uniformly on compact subsets of $\mathbb{D}$. Consequently, for any $\varepsilon>0$ there exists $n_{0}$ such that for $n \geq n_{0}$ the zeros of $s_{n, m_{n}, 1}$ and $s_{n, m_{n}, 2}$ are in $\{z:|z| \geq 1-\varepsilon\}$ and $\{z:|z| \leq 1+\varepsilon\}$, respectively.

Proof. The proof is basically the same as the one above. Fix a compact subset $K$ of $\mathbb{C} \backslash \overline{\mathbb{D}}$. Take the circle $\gamma_{R}$ in Lemma प of radius $R$ sufficiently close to 1 so that $K$ lies in the unbounded component of the complement of $\gamma_{R}$. Using (35), there exists $n_{0}(\varepsilon)$, such that for all $n \geq n_{0}(\varepsilon)$ and $z \in K$,

$$
\left|g_{n}(z)\right| \leq \frac{C_{1}}{|z|^{n}}, \quad \inf _{\zeta \in \gamma_{R}}\left|g_{n}(\zeta)\right| \geq C_{2} \frac{1}{R^{n}}
$$

where $C_{1}, C_{2}$ are positive constants. The representation (22) yields

$$
\left|s_{n, m, 2}(z) g_{n}(z)-1\right| \leq C\left(\frac{|R|}{|z|}\right)^{n-m_{n}+1},
$$

where $C$ is a constant. Letting $n \rightarrow \infty$ shows (40). The property of the zeros of $s_{n, m_{n}, 2}$ now follows from the Hurwitz theorem. Formula (41) and the property of the zeros of $s_{n, m_{n}, 1}$ are shown similarly.

The next result is the key to all subsequent developments in the paper. 
Theorem 2. Let $\sigma \in \operatorname{Reg} \mathbb{T}$. Then, for each compact set $K$,

$$
\limsup _{n \rightarrow \infty}\left\|s_{n, m_{n}} g_{n}-1\right\|_{K}^{1 / n} \leq \begin{cases}\|1 / z\|_{K}^{1-\bar{\rho}}, & K \subset \overline{\mathbb{C}} \backslash \overline{\mathbb{D}}, \\ \|z\| \frac{\rho}{K}, & K \subset \mathbb{D} .\end{cases}
$$

If $0<\underline{\rho} \leq \bar{\rho}<1$, then for any $\varepsilon>0$ there exists $n_{0}$, such that for $n \geq n_{0}$ the zeros of $s_{n, m_{n}}$ are in the annulus $\mathbb{A}_{\varepsilon}=\{z \in \mathbb{C}: 1-\varepsilon \leq|z| \leq 1+\varepsilon\}$, and

$$
\lim _{n \rightarrow \infty}\left|s_{n, m_{n}}(z)\right|^{1 / n}= \begin{cases}|z|, & z \in \mathbb{C} \backslash \overline{\mathbb{D}}, \\ 1, & z \in \mathbb{D}\end{cases}
$$

uniformly on compact subsets of the indicated regions.

Proof. As before, we write $m$ instead of $m_{n}$. Fix a compact subset $K$ of $\overline{\mathbb{C}} \backslash \overline{\mathbb{D}}$. By the triangle inequality

$$
\left\|s_{n, m} g_{n}-1\right\|_{K} \leq\left\|s_{n, m, 2} g_{n}-1\right\|_{K}+\left\|s_{n, m, 1} g_{n}\right\|_{K} .
$$

Using (37), the first term in the right-hand side of this inequality has $n$th root asymptotic behavior as $n$ goes to $\infty$ equal to the right-hand side of (42). To conclude the proof it is sufficient to show that the second term also has the same $n$th root asymptotic behavior as $n$ tends to $\infty$.

As mentioned, $s_{n, m, 1}^{*}(z)=z^{m} \overline{s_{n, m, 1}(1 / \bar{z})}$; then, using the second relation in (16), we get

$$
s_{n, m, 1}(z) g_{n}(z)=\frac{s_{n, m, 1}(z)}{s_{n, m, 1}^{*}(z)} \frac{z^{m-n}}{-\tau_{n}} \overline{s_{n, m, 1}(1 / \bar{z})} \overline{g_{n}(1 / \bar{z})} .
$$

Since $z \in K, 1 / \bar{z}$ lies in a compact subset of $\mathbb{D}$ and, according to (38), the product of the last two functions in the right-hand side converges uniformly to 1 for $z \in K$. Hence,

$$
\lim _{n \rightarrow \infty}\left\|s_{n, m, 1} g_{n}\right\|_{K}^{1 / n}=1
$$

Writing $s_{n, m, 1}(z)=c_{n} \prod_{k=1}^{m}\left(z-z_{n, k}\right)$, we have the expression

$$
\frac{s_{n, m, 1}(z)}{s_{n, m, 1}^{*}(z)}=\frac{c_{n}}{\overline{c_{n}}} \prod_{k=1}^{m} \frac{z-z_{n, k}}{1-\overline{z_{n, k}} z}
$$

According to the last assertion of Lemma 9, given $\varepsilon>0$ there exists $n_{0}(\varepsilon)$, such that for all $n \geq n_{0}$ all the zeros of $s_{n, m, 1}$ are of absolute value greater than $1-\varepsilon$. We take $\varepsilon$ sufficiently small so that $1 / \overline{z_{n, k}} \notin K$. In the rest of the proof we restrict our attention to such values of $n$. If $\left|z_{n, k}\right| \geq 1$, it is well known and easy to verify, that

$$
\left|\frac{z-z_{n, k}}{1-\overline{z_{n, k}} z}\right| \leq 1, \quad z \in K
$$

Let us consider the remaining $z_{n, k}$ for which $1-\varepsilon \leq\left|z_{n, k}\right|<1$. Then,

$$
\left|1 / \overline{z_{n, k}}\right| \leq(1-\varepsilon)^{-1}
$$

and

$$
\left|1 / \overline{z_{n, k}}-z_{n, k}\right| \leq(1-\varepsilon)^{-1}-(1-\varepsilon)=\varepsilon(2-\varepsilon) /(1-\varepsilon)
$$


(the points $1 / \overline{z_{n, k}}, z_{n, k}$ are symmetric with respect to $\mathbb{T}$ ). Without loss of generality, we may assume that $\varepsilon$ is chosen so that

$$
\inf _{z \in K}|z| \geq\left(1+\varepsilon^{1 / 2}(2-\varepsilon)\right) /(1-\varepsilon) \text {. }
$$

Hence,

$$
\inf _{z \in K}\left|z-1 / \bar{z}_{n, k}\right| \geq \inf _{z \in K}|z|-\left|1 / \bar{z}_{n, k}\right| \geq \varepsilon^{1 / 2}(2-\varepsilon) /(1-\varepsilon) .
$$

For such points $z_{n, k}$ and selection of $\varepsilon$, it follows that

$$
\left|\frac{z-z_{n, k}}{1-\overline{z_{n, k}} z}\right| \leq \frac{1}{\left|\overline{z_{n, k}}\right|} \frac{\left|z-1 / \overline{z_{n, k}}\right|+\left|1 / \overline{z_{n, k}}-z_{n, k}\right|}{\left|z-1 / \overline{z_{n, k}}\right|} \leq \frac{1+\varepsilon^{1 / 2}}{1-\varepsilon} .
$$

Putting all of this together, we find

$$
\left\|\frac{s_{n, m, 1}}{s_{n, m, 1}^{*}}\right\|_{K} \leq\left(\frac{1+\varepsilon^{1 / 2}}{1-\varepsilon}\right)^{m},
$$

which implies that

$$
\limsup _{n \rightarrow \infty}\left\|\frac{s_{n, m, 1}}{s_{n, m, 1}^{*}}\right\|_{K}^{1 / n} \leq\left(\frac{1+\varepsilon^{1 / 2}}{1-\varepsilon}\right)^{\bar{\rho}} .
$$

Letting $\varepsilon \rightarrow 0$, we obtain

$$
\limsup _{n \rightarrow \infty}\left\|\frac{s_{n, m, 1}}{s_{n, m, 1}^{*}}\right\|_{K}^{1 / n} \leq 1
$$

To conclude this part of the proof, using (44)-(46), it follows that

$$
\limsup _{n \rightarrow \infty}\left\|s_{n, m, 1} g_{n}\right\|_{K}^{1 / n} \leq\|1 / z\|_{K}^{1-\bar{\rho}}
$$

as needed.

The case of compact subsets $K \subset \mathbb{D}$ is treated analogously. We start with

$$
\left\|s_{n, m} g_{n}-1\right\|_{K} \leq\left\|s_{n, m, 1} g_{n}-1\right\|_{K}+\left\|s_{n, m, 2} g_{n}\right\|_{K}
$$

The first term in the right-hand side behaves correctly according to (38). To show that the second term follows the same asymptotics, we define $s_{n, m, 2}^{*}(z)=$ $z^{n} \overline{s_{n, m, 2}(1 / \bar{z})}$ and, due to the second relation in (16), we obtain

$$
s_{n, m, 2}(z) g_{n}(z)=\frac{s_{n, m, 2}(z)}{s_{n, m, 2}^{*}(z)} \frac{\overline{s_{n, m, 2}(1 / \bar{z})} \overline{g_{n}(1 / \bar{z})}}{-\tau_{n}} .
$$

From (37), we have

$$
\limsup _{n \rightarrow \infty}\left\|s_{n, m, 2} g_{n}\right\|_{K}^{1 / n}=1,
$$

and all we need to show is that

$$
\limsup _{n \rightarrow \infty}\left\|\frac{s_{n, m, 2}}{s_{n, m, 2}^{*}}\right\|_{K}^{1 / n} \leq\|z\| \frac{\rho}{K} .
$$

Indeed, this is the case since $s_{n, m, 2}(z)=z^{m+1} \prod_{k=1}^{n-m-1}\left(z-z_{n, k}\right)$ has a zero at $z=0$ of multiplicity at least $m+1$ and, therefore,

$$
\frac{s_{n, m, 2}(z)}{s_{n, m, 2}^{*}(z)}=z^{m+1} \prod_{k=1}^{n-m-1} \frac{z-z_{n, k}}{1-\overline{z_{n, k}} z} .
$$


The first factor in the right-hand side gives us

$$
\lim _{n \rightarrow \infty}\left\|z^{m+1}\right\|_{K}^{1 / n} \leq\|z\| \frac{\rho}{K}
$$

and one can show that

$$
\limsup _{n \rightarrow \infty}\left\|\prod_{k=1}^{n-m-1} \frac{z-z_{n, k}}{1-\overline{z_{n, k}} z}\right\|_{K}^{1 / n} \leq 1
$$

in a similar fashion as we did above with the other Blaschke product.

To conclude, when $0<\rho \leq \bar{\rho}<1$, (42) implies that

$$
\lim _{n \rightarrow \infty} s_{n, m_{n}}(z) g_{n}(z)=1, \quad \lim _{n \rightarrow \infty}\left|s_{n, m_{n}}(z) g_{n}(z)\right|^{1 / n}=1,
$$

uniformly on compact subsets of $\mathbb{C} \backslash \mathbb{T}$. The first one of these limits and the Hurwitz theorem give the statement concerning the zeros of $s_{n, m}$. The second limit together with (27) imply (43).

The following theorem is the analogue of Theorem 2 under the assumptions of Lemma 10. The proof is similar to that of Theorem 2 and therefore is omitted.

Theorem 3. Let $\sigma \in \mathbf{S}$ and $\lim _{n \rightarrow \infty} m_{n}=\lim _{n \rightarrow \infty} n-m_{n}=\infty$. Then,

$$
\lim _{n \rightarrow \infty} s_{n, m_{n}}(z) g_{n}(z)=1 \text {, }
$$

uniformly on compact subsets of $\overline{\mathbb{C}} \backslash \mathbb{T}$. Consequently, for any $\varepsilon>0$ there exists $n_{0}$, such that for $n \geq n_{0}$ the zeros of $s_{n, m_{n}}$ are in the annulus $\mathbb{A}_{\varepsilon}=\{z \in \mathbb{C}: 1-\varepsilon \leq$ $|z| \leq 1+\varepsilon\}$.

Theorem 2 shows that the zeros of the Stieltjes-type polynomials may only accumulate on $\mathbb{T}$ when $\sigma \in \operatorname{Reg} \mathbb{T}, \rho>0, \bar{\rho}<1$. This assertion is complemented by Theorem 4 below, whose formulation requires some additional definitions.

It is well known (see [24], Section 3.3) that among all probability measures $\lambda$ supported on a compact set $K$, there is a probability measure $\lambda_{K}$ (which is unique if cap $K>0$ ) with support in $K$, called the extremal or equilibrium measure of $K$, which minimizes the energy

$$
\mathcal{I}(\nu)=\iint \log \frac{1}{|z-t|} d \lambda(z) d \lambda(t) .
$$

Let $P(\lambda ; z)=-\int \log |z-t| d \lambda(t)$ be the potential of the measure $\lambda$. There exists a constant $F$, called the equilibrium constant of $K$, such that

$$
\left\{\begin{array}{l}
P\left(\lambda_{K} ; z\right) \leq F, \quad z \in \mathbb{C}, \\
P\left(\lambda_{K} ; z\right)=F, \quad z \in K \backslash A \quad \text { with } \quad \operatorname{cap} A=0 .
\end{array}\right.
$$

It may be shown that (48) characterizes the equilibrium measure and that the equilibrium constant $F$ is precisely the minimal energy $\mathcal{I}\left(\lambda_{K}\right)$. We also recall that cap $K=\exp \{-F\}$.

If we take $K=\mathbb{T}$, then $F=0$ and $\lambda_{\mathbb{T}}=d \theta /(2 \pi)$, since

$$
P(d \theta /(2 \pi) ; z)=\frac{1}{2 \pi} \int_{0}^{2 \pi} \frac{1}{\log \mid z-e^{i \theta \mid}} d \theta= \begin{cases}-\log |z|, & |z| \geq 1, \\ 0, & |z|<1,\end{cases}
$$

which solves the equilibrium problem (48) for $K=\mathbb{T}$. 
Let $\rho_{n}$ and $\rho$ be finite Borel measures on $\overline{\mathbb{C}}$. By $\rho_{n} \stackrel{*}{\longrightarrow} \rho, n \rightarrow \infty$, we denote the weak* convergence of $\rho_{n}$ to $\rho$ as $n$ tends to infinity. This means that for every continuous function $f$ on $\overline{\mathbb{C}}$,

$$
\lim _{n \rightarrow \infty} \int f(x) d \rho_{n}(x)=\int f(x) d \rho(x) .
$$

For a given polynomial $T$, we denote by $\Theta_{T}$ the normalized zero counting measure of $T$; that is,

$$
\Theta_{T}=\frac{1}{\operatorname{deg} T} \sum_{\xi: T(\xi)=0} \delta_{\xi} .
$$

The sum is taken over all the zeros of $T$ and $\delta_{\xi}$ denotes the Dirac measure concentrated at $\xi$.

Theorem 4. Let $\sigma \in \mathbf{R e g} \mathbb{T}$ and $0<\underline{\rho} \leq \bar{\rho}<1$. Then

$$
\Theta_{S_{n, m_{n}}} \stackrel{*}{\longrightarrow} \frac{d \theta}{2 \pi}, \quad n \rightarrow \infty .
$$

Proof. Set $\Theta_{S_{n, m_{n}}} \equiv \Theta_{n}$. All the measures $\Theta_{n}$ are probability measures. Let $\Delta \subset \mathbb{N}$ be a subsequence of indices, such that

$$
\Theta_{n} \stackrel{*}{\longrightarrow} \Theta, \quad n \in \Delta, \quad n \rightarrow \infty .
$$

It suffices to show that $\Theta \equiv \lambda_{\mathbb{T}}$ for any such sequence $\Delta$ of indices. We also may assume that the sequence $m_{n} / n$ is convergent when $n \in \Delta$.

According to Theorem 2 , the support of $\Theta$ is contained in $\mathbb{T}$. Taking (43) and (25) into account, we have that

$$
\lim _{n \in \Delta} P\left(\Theta_{n} ; z\right)=-\lim _{n \in \Delta} \frac{1}{n} \log \left|S_{n, m_{n}}\right|=P\left(\lambda_{\mathbb{T}} ; z\right),
$$

uniformly on compact subsets of $\mathbb{C} \backslash \mathbb{T}$. On the other hand, from (49) one obtains

$$
\lim _{n \in \Delta} P\left(\Theta_{n} ; z\right)=P(\Theta ; z)
$$

uniformly on compact subsets of $\mathbb{C} \backslash \mathbb{T}$. Thus, $P(\Theta ; z)=P\left(\lambda_{\mathbb{T}} ; z\right)$ except for a set of Lebesgue measure zero in the complex plane; therefore, from Theorem 3.7.4 in 24, we obtain that $\Theta \equiv \lambda_{\mathbb{T}}$ as we wanted to show.

For the other two classes of measures, we have the following result.

Corollary 1. If $\sigma$ is such that $\lim _{n \rightarrow \infty} \Phi_{n}(0)=0$ and $0<\underline{\rho} \leq \bar{\rho}<1$, then

$$
\lim _{n \rightarrow \infty} \frac{s_{n+1, m_{n}}(z)}{s_{n, m_{n}}(z)}= \begin{cases}z, & z \in \mathbb{C} \backslash \overline{\mathbb{D}}, \\ -1, & z \in \mathbb{D},\end{cases}
$$

uniformly on compact subsets of the indicated regions. When $\sigma \in \mathbf{S}$, and $\lim _{n \rightarrow \infty} m_{n}$ $=\lim _{n \rightarrow \infty} n-m_{n}=\infty$, we obtain

$$
\lim _{n \rightarrow \infty} \overline{\tau_{n}} s_{n, m_{n}}(z)=-S_{\sigma}^{-1}(z),
$$

uniformly on compact subsets of $\mathbb{D}$ and

$$
\lim _{n \rightarrow \infty} \frac{s_{n, m_{n}}(z)}{z^{n}}=\frac{1}{\overline{S_{\sigma}(1 / \bar{z})}}=S_{\sigma}(z),
$$

uniformly on compact subsets of $\mathbb{C} \backslash \overline{\mathbb{D}}$. 
Proof. We write again $m$ instead of $m_{n}$ throughout the proof. Using (42) or (47), depending on the assumptions, we have

$$
\lim _{n \rightarrow \infty} s_{n, m}(z) g_{n}(z)=1,
$$

uniformly on compact subsets of $\overline{\mathbb{C}} \backslash \mathbb{T}$. For the proof of (50), notice that

$$
\lim _{n \rightarrow \infty} \frac{s_{n+1, m}(z)}{s_{n, m}(z)}=\lim _{n \rightarrow \infty} \frac{s_{n+1, m}(z) g_{n+1}(z)}{s_{n, m}(z) g_{n}(z)} \frac{g_{n}(z)}{g_{n+1}(z)}=\lim _{n \rightarrow \infty} \frac{g_{n}(z)}{g_{n+1}(z)},
$$

and we only have to use (32) to conclude what we need. For (51), since

$$
\lim _{n \rightarrow \infty} \overline{\tau_{n}} s_{n, m}(z)=\lim _{n \rightarrow \infty} s_{n, m}(z) g_{n}(z) \frac{1}{\tau_{n} g_{n}(z)}=\lim _{n \rightarrow \infty} \frac{1}{\tau_{n} g_{n}(z)},
$$

use (36), whereas to demonstrate (52), write

$$
\lim _{n \rightarrow \infty} \frac{s_{n, m}(z)}{z^{n}}=\lim _{n \rightarrow \infty} s_{n, m}(z) g_{n}(z) \frac{1}{z^{n} g_{n}(z)}=\lim _{n \rightarrow \infty} \frac{1}{z^{n} g_{n}(z)}
$$

and apply (35).

\section{Convergence of Quadrature formulae}

Fix $n \in \mathbb{N}, \tau_{n} \in \mathbb{T}$, and $m \in\{0,1, \ldots, n-1\}$. Let $L_{2 n, m}$ be the polynomial of degree at most $2 n$ given by

$$
\frac{L_{2 n, m}(z)}{z^{n}}=\int \frac{\zeta+z}{\zeta-z}\left(\frac{s_{n, m}(z) w_{n}^{*}(z)}{z^{n}}-\frac{s_{n, m}(\zeta) w_{n}^{*}(\zeta)}{\zeta^{n}}\right) d \sigma(\zeta) .
$$

We denote by

$$
F_{\sigma}(z)=\int \frac{\zeta+z}{\zeta-z} d \sigma(\zeta)
$$

the Carathéodory function associated with the measure $\sigma$ and define

$$
R_{2 n, m}(z)=\frac{L_{2 n, m}(z)}{s_{n, m}(z) w_{n}^{*}(z)}
$$

Theorem 5. Assume that either $\sigma \in \boldsymbol{R e g} \mathbb{T}$ and $0<\rho \leq \bar{\rho}<1$, or $\sigma \in \mathbf{S}$ and $\lim _{n \rightarrow \infty} m_{n}=\lim _{n \rightarrow \infty} n-m_{n}=\infty$. Then, for each compact set $K$,

$$
\limsup _{n \rightarrow \infty}\left\|F_{\sigma}-R_{2 n, m_{n}}\right\|_{K}^{1 / n} \leq \begin{cases}\|1 / z\|_{K}^{2-\bar{\rho}}, & K \subset \overline{\mathbb{C}} \backslash \overline{\mathbb{D}}, \\ \|z\|_{K}^{1+\underline{\rho}}, & K \subset \mathbb{D} .\end{cases}
$$

Proof. Formula (53) may be expressed as

$$
s_{n, m}(z) w_{n}^{*}(z) F_{\sigma}(z)-L_{2 n, m}(z)=z^{n} \int \frac{\zeta+z}{\zeta-z} \frac{s_{n, m}(\zeta) w_{n}^{*}(\zeta)}{\zeta^{n}} d \sigma(\zeta) .
$$

Since $(\zeta+z) /(\zeta-z)=1+2 z /(\zeta-z)$, using the identity $\overline{w_{n}(\zeta)}=w_{n}^{*}(\zeta) / \zeta^{n}$ and orthogonality, we have

$$
\begin{aligned}
s_{n, m}(z) w_{n}^{*}(z) F_{\sigma}(z)-L_{2 n, m}(z) & =-2 z^{n} \int \frac{s_{n, m}(\zeta) \overline{w_{n}(\zeta)}}{1-\zeta / z} d \sigma(\zeta) \\
& =2 z^{n}\left(1-s_{n, m}(z) g_{n}(z)\right)
\end{aligned}
$$


for $z \notin \mathbb{T}$, where we have used (21) in the second equality. We may rewrite (55) as

$$
F_{\sigma}(z)-R_{2 n, m}(z)=\frac{2 z^{n} g_{n}(z)}{s_{n, m}(z) g_{n}(z) w_{n}^{*}(z)}\left(1-s_{n, m}(z) g_{n}(z)\right), \quad z \notin \mathbb{T} .
$$

Fix a compact set $K$ contained either in $\mathbb{D}$ or $\overline{\mathbb{C}} \backslash \overline{\mathbb{D}}$ and choose $\varepsilon>0$ sufficiently small, so that the annulus $\mathbb{A}_{\varepsilon}=\{z \in \mathbb{C}: 1-\varepsilon \leq|z| \leq 1+\varepsilon\}$ does not intersect $K$. According to Theorem 2 or 3 . depending on the hypothesis, there exists $n_{0}$, such that for all $n \geq n_{0}$ all the zeros of $s_{n, m}$ lie in $\mathbb{A}_{\varepsilon}$. We will only consider such values of $n$ and recall that $m=m_{n}$.

Using the definition of $w_{n}^{*}$, it is easy to verify that (26) is equivalent to

$$
\lim _{n \rightarrow \infty}\left|w_{n}^{*}(z)\right|^{1 / n}= \begin{cases}|z|, & z \in \mathbb{C} \backslash \overline{\mathbb{D}}, \\ 1, & z \in \mathbb{D},\end{cases}
$$

uniformly on compact subsets of the indicated regions. On the basis of the error formula (56), (54) follows directly from (27), (42), (57), and (47) (when working with the second group of assumptions).

Since the zeros of $w_{n}$ are simple and lie on $\mathbb{T}$, the same is true for the zeros of $w_{n}^{*}$. Regarding the zeros of $s_{n, m}$, all we know is that they accumulate on $\mathbb{T}$ under appropriate assumptions, so they may be multiple, coincide with some zeros of $w_{n}^{*}$, or lie outside $\mathbb{T}$. Nonetheless (see Lemma 1) $s_{n, m}(0)=-\tau_{n} /\left\|\Phi_{n}\right\| \neq 0$. Therefore, we have

$$
W_{n}(z) S_{n, m}(z)=\prod_{i=1}^{M}\left(z-z_{n, i}\right)^{\alpha_{i}}, \quad \alpha_{1}+\cdots+\alpha_{M}=2 n,
$$

where $z_{n, i} \neq 0$ for $i=1, \ldots, M$, and $W_{n}$ is $w_{n}^{*}$ normalized to be monic.

Lemma 11. The rational function $R_{2 n, m}$ admits the representation

$$
R_{2 n, m}(z)=\sum_{i=1}^{M} \sum_{j=0}^{\alpha_{i}-1} \lambda_{i, j} g_{z}^{(j)}\left(z_{n, i}\right)
$$

where $g_{z}(w)=(w+z) /(w-z)$.

Proof. Consider the following decomposition into simple fractions

$$
\frac{R_{2 n, m}(z)}{2 z}=\frac{a}{2 z}-\sum_{i=1}^{M} \sum_{j=0}^{\alpha_{i}-1} \frac{j ! \lambda_{i, j}}{\left(z-z_{n, i}\right)^{j+1}} .
$$

Certainly, $a, M, \alpha_{i}$, and $\lambda_{i, j}$ depend on $n$ and $m$. However, since for the time being these indices remain fixed, we will for notational simplicity omit indicating this dependence.

Set $h_{z}(w):=1 /(z-w)$. Taking derivatives with respect to $w$, we find that $h_{z}^{(j)}(w)=j ! /(z-w)^{j+1}$. With this notation, the previous formula may be written as

$$
R_{2 n, m}(z)=a-2 z \sum_{i=1}^{M} \sum_{j=0}^{\alpha_{i}-1} \lambda_{i, j} h_{z}^{(j)}\left(z_{n, i}\right) .
$$

In particular, $R_{2 n, m}(0)=a$ and $R_{2 n, m}(\infty)=a-2 \sum_{i=1}^{M} \lambda_{i, 0}$. 
The interpolation conditions satisfied by $R_{2 n, m}$ with respect to $F_{\sigma}$ at 0 and $\infty$ imply that $R_{2 n, m}(0)=F_{\sigma}(0)$ and $R_{2 n, m}(\infty)=F_{\sigma}(\infty)$; therefore,

$$
R_{2 n, m}(0)+R_{2 n, m}(\infty)=2\left(a-\sum_{i=1}^{M} \lambda_{i, 0}\right)=F_{\sigma}(0)+F_{\sigma}(\infty)=0 .
$$

It follows that

$$
a=\sum_{i=1}^{M} \lambda_{i, 0}
$$

Notice that $g_{z}(w)=1-2 z h_{z}(w)$. Taking derivatives with respect to $w$, we obtain $g_{z}^{(j)}(w)=-2 z h_{z}^{(j)}(w), j \geq 1$. With this notation and (58), we obtain

$$
\begin{aligned}
R_{2 n, m}(z) & =a-2 z \sum_{i=1}^{M} \lambda_{i, 0} h_{z}\left(z_{n, i}\right)-2 z \sum_{i=1}^{M} \sum_{j=1}^{\alpha_{i}-1} \lambda_{i, j} h_{z}^{(j)}\left(z_{n, i}\right) \\
& =a-\sum_{i=1}^{M} \lambda_{i, 0}\left(1-g_{z}\left(z_{n, i}\right)\right)+\sum_{i=1}^{M} \sum_{j=1}^{\alpha_{i}-1} \lambda_{i, j} g_{z}^{(j)}\left(z_{n, i}\right) \\
& =\sum_{i=1}^{M} \sum_{j=0}^{\alpha_{i}-1} \lambda_{i, j} g_{z}^{(j)}\left(z_{n, i}\right) .
\end{aligned}
$$

Define the quadrature formula

$$
\widetilde{I}_{2 n}(f)=\sum_{i=1}^{M} \sum_{j=0}^{\alpha_{i}-1} \lambda_{i, j} f^{(j)}\left(z_{n, i}\right)
$$

determined by the representation of $R_{2 n, m}$ in Lemma 11 Let $\gamma_{r}=\{z:|z|=r\}$. We also denote the corresponding circle with positive orientation by $\gamma_{r}$.

Theorem 6. Let $f$ be analytic in $\mathbb{A}=\left\{z: r_{1}<|z|<r_{2}\right\}, r_{1}<1<r_{2}$. Suppose that the zeros $z_{n, i}, i=1, \ldots, M$, of $W_{n} S_{n, m}$ lie in $\mathbb{A}$. Then

$$
I_{\sigma}(f)-\widetilde{I}_{2 n}(f)=-\frac{1}{2 \pi i} \int_{\gamma}\left(F_{\sigma}(\zeta)-R_{2 n, m}(\zeta)\right) \frac{f(\zeta)}{2 \zeta} d \zeta,
$$

where $\gamma=\gamma_{r_{2}^{\prime}}-\gamma_{r_{1}^{\prime}}, r_{1}<r_{1}^{\prime}<|z|<r_{2}^{\prime}<r_{2}$ and $r_{1}^{\prime}<\left|z_{n, i}\right|<r_{2}^{\prime}$.

Proof. Let $f$ be analytic in the annulus $\mathbb{A}$. From Cauchy's integral formula, for all $z$ with $r_{1}<r_{1}^{\prime}<|z|<r_{2}^{\prime}<r_{2}$, we have

$$
f(z)=\frac{1}{2 \pi i} \int_{\gamma} \frac{f(\zeta)}{\zeta-z} d \zeta=\frac{1}{2 \pi i} \int_{\gamma} \frac{2 \zeta}{\zeta-z} \frac{f(\zeta)}{2 \zeta} d \zeta=\frac{1}{2 \pi i} \int_{\gamma} \frac{\zeta+z}{\zeta-z} \frac{f(\zeta)}{2 \zeta} d \zeta,
$$

where $\gamma=\gamma_{r_{2}^{\prime}}-\gamma_{r_{1}^{\prime}}$. Consequently, using Fubini's theorem,

$$
I_{\sigma}(f)=\int\left(\frac{-1}{2 \pi i} \int_{\gamma} \frac{z+\zeta}{z-\zeta} \frac{f(\zeta)}{2 \zeta} d \zeta\right) d \sigma(z)=-\frac{1}{2 \pi i} \int_{\gamma} F_{\sigma}(\zeta) \frac{f(\zeta)}{2 \zeta} d \zeta .
$$

From (61), we also obtain

$$
f^{(j)}(z)=-\frac{1}{2 \pi i} \int_{\gamma} g_{\zeta}^{(j)}(z) \frac{f(\zeta)}{2 \zeta} d \zeta, \quad j \geq 0,
$$

for all $z$ with $r_{1}^{\prime}<|z|<r_{2}^{\prime}$. 
Since the zeros of $S_{n, m}$ lie in the annulus $\left\{z: r_{1}^{\prime}<|z|<r_{2}^{\prime}\right\}$, on account of (63) and the representation of $R_{2 n, m}$ in Lemma 11, we obtain

$$
\begin{aligned}
\widetilde{I}_{2 n}(f) & =\sum_{i=1}^{M} \sum_{j=0}^{\alpha_{i}-1} \lambda_{i, j} \frac{-1}{2 \pi i} \int_{\gamma} g_{\zeta}^{(j)}\left(z_{n, k}\right) \frac{f(\zeta)}{2 \zeta} d \zeta \\
& =\frac{-1}{2 \pi i} \int_{\gamma} R_{2 n, m}(\zeta) \frac{f(\zeta)}{2 \zeta} d \zeta .
\end{aligned}
$$

Finally, formula (60) is the difference between (62) and (64).

Corollary 2. The quadrature rule (59) is exact in $\Lambda_{n+m, 2 n-m-1}$ and therefore coincides with the interpolatory rule $I_{2 n}$ considered in Theorem 1 .

Proof. Combining the first equality in (55) and (14), with $L(z)=z^{n-m}$ and $L(z)=$ $1 / z^{m}$, it follows that

$$
F_{\sigma}(z)-R_{2 n, m}(z)=\frac{-2 z^{m}}{s_{n, m}(z) w_{n}^{*}(z)} \int \frac{\zeta^{n-m} s_{n, m}(\zeta) \overline{w_{n}(\zeta)}}{1-\zeta / z} d \sigma(\zeta)
$$

and

$$
F_{\sigma}(z)-R_{2 n, m}(z)=\frac{-2 z^{n+m+1}}{s_{n, m}(z) w_{n}^{*}(z)} \int \frac{s_{n, m}(\zeta) \overline{w_{n}(\zeta)}}{\zeta^{m}(z-\zeta)} d \sigma(\zeta)
$$

Therefore (recall that $s_{n, m}(0) w_{n}^{*}(0) \neq 0$ ),

$$
F_{\sigma}(z)-R_{2 n, m}(z)= \begin{cases}\mathcal{O}\left(z^{m-2 n}\right), & z \rightarrow \infty \\ \mathcal{O}\left(z^{n+m+1}\right), & z \rightarrow 0\end{cases}
$$

Consequently, if $L \in \Lambda_{n+m, 2 n-m-1}$, then

$$
\left(F_{\sigma}(z)-R_{2 n, m}(z)\right) \frac{L(z)}{2 z}= \begin{cases}\mathcal{O}\left(1 / z^{2}\right), & z \rightarrow \infty \\ \mathcal{O}(1), & z \rightarrow 0\end{cases}
$$

The Laurent polynomial $L$ is analytic in $\mathbb{C} \backslash\{0\}$. We may apply Theorem 6 with $r_{1}=0$ and $r_{2}=+\infty$ and choose $r_{1}^{\prime}$ and $r_{2}^{\prime}$ sufficiently small and large, respectively, so that the zeros of $S_{n, m}$ belong to the set $\left\{z: r_{1}^{\prime}<|z|<r_{2}^{\prime}\right\}$. Then, formula (60) implies

$$
\begin{aligned}
I_{\sigma}(L)-\widetilde{I}_{2 n}(L) & =-\frac{1}{2 \pi i} \int_{\gamma_{r_{2}^{\prime}}}\left(F_{\sigma}(\zeta)-R_{2 n, m}(\zeta)\right) \frac{L(\zeta)}{2 \zeta} d \zeta \\
& +\frac{1}{2 \pi i} \int_{\gamma_{r_{1}^{\prime}}}\left(F_{\sigma}(\zeta)-R_{2 n, m}(\zeta)\right) \frac{L(\zeta)}{2 \zeta} d \zeta
\end{aligned}
$$

Taking into consideration (65) and the fact that $\left(F_{\sigma}(z)-R_{2 n, m}(z)\right) L(z) / 2 z$ is analytic in open neighborhoods of $\left\{z:|z| \leq r_{1}^{\prime}\right\}$ and $\left\{z:|z| \geq r_{2}^{\prime}\right\}$, we obtain from Cauchy's integral formula applied in those open sets that

$$
\int_{\gamma_{r_{1}^{\prime}}}\left(F_{\sigma}(\zeta)-R_{2 n, m}(\zeta)\right) \frac{L(\zeta)}{2 \zeta} d \zeta=\int_{\gamma_{r_{2}^{\prime}}}\left(F_{\sigma}(\zeta)-R_{2 n, m}(\zeta)\right) \frac{L(\zeta)}{2 \zeta} d \zeta=0,
$$

which, together with (66), gives the result. 
Theorem 7. Let $f$ be analytic in $\mathbb{A}=\left\{z: r_{1}<|z|<r_{2}\right\}, r_{1}<1<r_{2}$. Assume that either $\sigma \in \mathbf{R e g} \mathbb{T}$ and $0<\underline{\rho} \leq \bar{\rho}<1$, or $\sigma \in \mathbf{S}$ and $\lim _{n \rightarrow \infty} m_{n}=\lim _{n \rightarrow \infty} n-m_{n}=$ $\infty$. Then

$$
\limsup _{n \rightarrow \infty}\left|I_{\sigma}(f)-I_{2 n}(f)\right|^{1 / n} \leq \max \left\{\left(1 / r_{2}\right)^{2-\bar{\rho}}, r_{1}^{1+\underline{\rho}}\right\}
$$

Proof. Fix $\varepsilon>0$. For all sufficiently large $n$, from (54), we have

$$
\begin{aligned}
& \left\|F_{\sigma}-R_{2 n, m_{n}}\right\|_{\gamma_{r_{2}^{\prime}}} \leq\left(r_{2}^{\prime}-\varepsilon\right)^{(\bar{\rho}-2) n}, \\
& \left\|F_{\sigma}-R_{2 n, m_{n}}\right\|_{\gamma_{r_{1}^{\prime}}} \leq\left(r_{1}^{\prime}+\varepsilon\right)^{(1+\underline{\rho}) n} .
\end{aligned}
$$

The zeros of $S_{n, m_{n}}$ tend to $\mathbb{T}$ due to either Theorem 2 or 3 , Using (60) and Corollary 2, it follows that

$$
\begin{aligned}
\left|I_{\sigma}(f)-I_{2 n}(f)\right| & \leq \frac{1}{2 \pi} \int_{\gamma_{r_{2}^{\prime}}}\left|\left(F_{\sigma}(\zeta)-R_{2 n, m_{n}}(\zeta)\right) \frac{f(\zeta)}{2 \zeta}\right||d \zeta| \\
& +\frac{1}{2 \pi} \int_{\gamma_{r_{1}^{\prime}}}\left|\left(F_{\sigma}(\zeta)-R_{2 n, m_{n}}(\zeta)\right) \frac{f(\zeta)}{2 \zeta}\right||d \zeta| \\
& \leq C\left[\left(r_{2}^{\prime}-\varepsilon\right)^{(\bar{\rho}-2) n}+\left(r_{1}^{\prime}+\varepsilon\right)^{(1+\underline{\rho}) n}\right],
\end{aligned}
$$

where $C$ is a constant that depends on $\|f(\zeta) /(2 \zeta)\|_{\gamma}$ and the length of $\gamma$ but is independent of $n$. Hence,

$$
\left|I_{\sigma}(f)-I_{2 n}(f)\right|^{1 / n} \leq(2 C)^{1 / n} \max \left\{\left(r_{2}^{\prime}-\varepsilon\right)^{(\bar{\rho}-2)},\left(r_{1}^{\prime}+\varepsilon\right)^{(1+\underline{\rho})}\right\} .
$$

Taking limits as $n$ goes to $\infty$ and then letting $\varepsilon \rightarrow 0, r_{2}^{\prime} \rightarrow r_{2}$, and $r_{1}^{\prime} \rightarrow r_{1}$, gives (67).

Suppose that we have taken a sequence $\left\{m_{n}\right\}, n \in \mathbb{N}$, such that $\bar{\rho}=\rho=\rho$ in the above theorem. Notice that the two elements under the maximum sign in the righthand side of (67) react oppositely as $\rho$ increases from 0 to 1 (for $r_{1}, r_{2}$ fixed). The first one increases with $\rho$ and the second one decreases. In the bound we have to take the largest of the two. Therefore, to obtain the best possible estimate, depending on the region where $f$ is analytic, we should choose the sequence $\left\{m_{n}\right\}, n \in \mathbb{N}$, appropriately.

When $r_{1} r_{2}=1$, the best possible estimate is obtained for $\rho=1 / 2$. When $r_{1} r_{2}<$ 1 we should interpolate more often at $\infty$ than at 0 . The contrary is appropriate when $r_{1} r_{2}>1$. The best possible choice of $\rho$ is such that $r_{1}^{1+\rho}=\left(1 / r_{2}\right)^{2-\rho}$; that is, $\rho=(2+\eta) /(1-\eta), \eta=\log r_{1} / \log r_{2}$. Unfortunately, this choice is not always possible with $\rho \in[0,1]$. Therefore, the rule is the following: take a sequence $\left\{m_{n}\right\}, n \in \mathbb{N}$, such that

$$
\rho=\left\{\begin{array}{cl}
0, & (2+\eta) /(1-\eta)<0, \\
(2+\eta) /(1-\eta), & (2+\eta) /(1-\eta) \in[0,1], \\
1, & (2+\eta) /(1-\eta)>1 .
\end{array}\right.
$$

The values $\rho=0$ and $\rho=1$ are only allowed if $\lim _{n \rightarrow \infty} m_{n}=\lim _{n \rightarrow \infty} n-m_{n}=\infty$ and $\sigma \in \mathbf{S}$. If this is not the case, then we must take values of $\rho$ close to 0 or 1 . 
In order to extend this theorem to non-analytic integrands, it is convenient to find (non-trivial) families of measures for which the zeros of the Stieltjes-type polynomials lie on $\mathbb{T}$ and are simple. This is a nice open problem. Nevertheless, we have found a number of examples for which either the corresponding Stieltjes-type polynomials satisfy such nice properties or there is numerical evidence of this good behavior.

The results of this section provide the foundation for the development of GaussKronrod-type quadrature rules, referred to as Szegö-Kronrod rules, for the integration of $2 \pi$-periodic functions. A few low-order Szegö-Kronrod rules can be found in [13. We are presently developing numerical methods for the determination of Szegö-Kronrod rules of arbitrary order. These methods along with the examples mentioned above will be presented in a forthcoming paper.

After this paper was submitted, we have learned that F. Peherstorfer 20] also has results on Szegö-Kronrod rules with good properties. His construction differs slightly from ours.

\section{REFERENCES}

[1] G. S. Ammar, D. Calvetti, and L. Reichel, Computation of Gauss-Kronrod quadrature rules with non-positive weights, Electron. Trans. Numer. Anal., 9 (1999), 26-38. MR 1749795 (2001c:65030)

[2] M. Bello Hernández, B. de la Calle Ysern, J. J. Guadalupe Hernández, and G. López Lagomasino, Asymptotics for Stieltjes polynomials, Padé approximants, and Gauss-Kronrod quadrature, J. Anal. Math., 86 (2002), 1-23. MR1894475 (2002m:41021)

[3] I. S. Berezin and N. P. Zhidkov, Computing Methods, Pergamon Press, Oxford, 1965.

[4] A. Bultheel, P. González-Vera, E. Hendriksen, and O. Nuåstad, On the convergence of multipoint Padé-type approximants and quadrature formulas associated with the unit circle, Numer. Algorithms, 13 (1996), 321-344. MR1430523 (97h:41029)

[5] B. De la Calle Ysern and F. Peherstorfer, Ultraspherical Stieltjes polynomials and Gauss-Kronrod quadrature behave nicely for $\lambda<0$, SIAM J. Numer. Anal., 45 (2007), 770 786. MR2300296 (2008d:33009)

6] D. Calvetti, G. H. Golub, W. B. GragG, and L. Reichel, Computation of Gauss-Kronrod quadrature rules, Math. Comp., 69 (2000), 1035-1052. MR1677474 (2000j:65035)

[7] S. EHRICH, On product integration with Gauss-Kronrod nodes, SIAM J. Numer. Anal., 35 (1998), 78-92. MR1618432(99e:65044)

[8] S. Ehrich And G. Mastroianni, Stieltjes polynomials and Lagrange interpolation, Math. Comp., 66 (1997), 311-331. MR.1388888 (97j:65013)

[9] W. Gautschi, Gauss-Kronrod quadrature - a survey, in Numerical Methods and Approximation Theory III, G. V. Milovanović, ed., University of Niš, Niš, 1988, 39-66. MR960329 (89k:41035)

[10] Ya. L. Geronimus, On the trigonometric moment problem, Ann. of Math. (2), 47 (1946), 742-761. MR0018265 (8:265d)

[11] P. González-Vera, O. NJåstad, And J. C. Santos-León, Some results about numerical quadrature on the unit circle, Adv. Comput. Math., 5 (1996), 297-328. MR1414284 (98f:41028)

[12] Ch. Hermite and T. J. Stieltues, Correspondance d'Hermite et de Stieltjes, Prentice-Hall, Englewood Cliffs, NJ, 1966.

[13] C. Jagels and L. Reichel, Szegö-Lobatto quadrature rules, J. Comput. Appl. Math., 200 (2007), 116-126. MR2276819 (2008c:65074)

[14] W. B. Jones, O. NJ̊̊stad, And W. J. Thron, Moment theory, orthogonal polynomials, quadrature, and continued fractions associated with the unit circle, Bull. London Math. Soc., 21 (1989), 113-152. MR 976057 (90e:42027)

[15] A. S. Kronrod, Nodes and weights for quadrature formulae. Sixteen-Place Tables, Nauka Moscow, 1964, English transl., Consultants Bureau, New York, 1965. MR0183116 (32:598) 
[16] D. P. Laurie, Calculation of Gauss-Kronrod quadrature rules, Math. Comp., 66 (1997), 1133-1145. MR:1422788 (98m:65030)

[17] A. Máté, P. NEVAI, AND V. TOTIK, Extensions of Szegö's theory of orthogonal polynomials, II, Constr. Approx., 3 (1987), 51-72. MR892168 (88m:42044a)

[18] G. Monegato, Stieltjes polynomials and related quadrature rules, SIAM Rev., 24 (1982), 137-158. MR652464 (83d:65067)

[19] G. Monegato, An overview of the computational aspects of Kronrod quadrature rules, Numer. Algorithms, 26 (2001), 173-196. MR1829797|(2002a:65051)

[20] F. Peherstorfer, Szegö-Kronrod quadrature on the unit circle, manuscript.

[21] F. Peherstorfer and K. Petras, Ultraspherical Gauss-Kronrod quadrature is not possible for $\lambda>3$, SIAM J. Numer. Anal., 37 (2000), 927-948. MR1749243 (2001g:33010)

[22] R. Piessens, E. de Doncker-Kapenga, C. W. Überhuber, and D. K. Kahaner, QUADPACK: A subroutine package for automatic integration, Springer Ser. Comput. Math., 1, Springer, Berlin, 1983. MR.712135 (85b:65022)

[23] E. A. Rakhmanov, On asymptotic properties of polynomials orthogonal on the circle with weights not satisfying Szego"'s condition, Math. USSR Sbornik, 58 (1987), 149-167. MR.854969 (88b:42033)

[24] T. Ransford, Potential Theory in the Complex Plane, London Mathematical Society, Student Texts 28, Cambridge University Press, Cambridge, 1995. MR.1334766 (96e:31001)

[25] B. Simon, Orthogonal Polynomials on the Unit Circle, AMS Colloquium Publications 54, Parts I, II, Providence, RI, 2005.

[26] H. Stahl and V. Totik, General Orthogonal Polynomials, Cambridge University Press, Cambridge, 1992. MR 1163828 (93d:42029)

[27] G. SzEGö, Über gewisse orthogonale Polynome, die zu einer oszillierenden Belegungsfunktion gehören, Math. Ann., 110 (1935), 501-513. MR1512952

Departamento de Matemática Aplicada, E. T. S. de Ingenieros Industriales, UniverSidad Politécnica de Madrid, José G. Abascal 2, 28006 Madrid, Spain

E-mail address: bcalle@etsii.upm.es

Departamento de Matemáticas, Escuela Politécnica Superior, Universidad Carlos III DE Madrid, Universidad 30, 28911 Leganés, Spain

E-mail address: lago@math.uc3m.es

Department of Mathematical Sciences, Kent State University, Kent, Ohio 44242

E-mail address: reichel@math.kent.edu 